\title{
Variability and heritability of some onion (Allium cepa L.) characteristics of parental forms, $F_{1}$ hybrids and $F_{2}$ generation
}

\section{ROCH W. DORUCHOWSKI}

\begin{abstract}
Department of Vegetable Breeding and Genetics, Research Institute of Vegetable Crops, u1. 22 Lipca 1/3, 96-100 Skierniewice, Poland
\end{abstract}

(Received: April 2, 1985)

\begin{abstract}
The object of the studies in 1977 was a population of $15 F_{1}$ hybrids, $11 F_{2}$ progenies and 16 parental forms (8 male-sterile $\mathrm{A}$ lines and 8 inbred $\mathrm{C}$ lines - Table 2). Variability and heritability of some onion characteristic weight, height diameter of the bulbs and their shape, collar thickness, adherence and color of dry outer skin, bulb firmness, shape of the collar and position of root disc) were studied. Variability and heritability estimates of bulb weight, diameter and shape of the collar were relatively high $(30-70 \%)$. Variability of the skin thickness was also high, but heritability of this trait was low. The lowest variability and heritability were shown for bulb firmness. The high heritability of some traits indicates the possibility of increasing the effects of selection and improvement, especially of the weight and shape of bulbs.
\end{abstract}

\section{INTRODUCTION}

Most onion characteristics are determined by polygenes. This makes quick progress in breeding difficult because improvement and stabilization of these traits requires many tedious and lengthy efforts.

Most onion traits have been the subject of observations and descriptions (Harada, 1961; Doruchowski, 1968; Dowker and Fennell, 1974; E l-Shafie and Ahmed, 1977; Kampe, 1967; M c Collu m, 1966, 1968; Schwe is g u th, 1978). However, there is still a lack of sufficient information about which of these traits are the most important in the selection of parental components for breeding varieties and $F_{1}$ hybrids. Attempts at even partially solving this problem can be useful in starting onion breeding in the proper direction towards improving important horticultural characteristics. 


\section{MATERIALS AND METHODS}

The study was done at the Research Institute of Vegetable Crops in Skierniewice in 1977 and 1978.

In 1977 , the following lines were studied: $15 F_{1}$ hybrids, $11 F_{2}$ hybrids and 16 parental components, that is, 8 maternal male-sterile lines $\mathrm{A}$ and 8 inbred paternal lines $\mathrm{C}$ (Table 2).

The male-sterile lines A were developed from the variety 'Wolska' and from generations resulting from the back-crossing of American male-sterile lines with lines from the varieties 'Dako' and 'Rawska'.

The paternal lines $\mathrm{C}$ were developed from the varieties 'Wolska', 'Kutnowska' and 'Rawska'. The lines have been maintained from 1962 by the use of partial inbreeding and recurring selection. The use of strict inbreeding of these lines was impossible due to the danger of depression (in $\mathrm{S}_{3}$ and later generations) expressing itself in the fall of viability of seeds and plants. For this reason, the parental lines were heterozygous in respect to many traits. They were well aqualized for several years as far as utilitarian traits are concerned.

In 1978,7 of the same $F_{1}$ hybrids as in 1977 were studied as well as 5 other $F_{1}$ hybrids, 12 parental components (Table 2), that is, 7 maternal lines $\mathrm{A}$ and 5 paternal lines $\mathrm{C}$.

Seeds of $F_{1}$ hybrids and parental lines were sown in hotbeds. The seedlings were transplanted to the field on May 4, 100 per plot $(4.80 \times 0.9 \mathrm{~m})$ having an area of $4.32 \mathrm{~m}^{2}$, in 3 replications, by the random block method. The rows were spaced $45 \mathrm{~cm}$ apart and the plants in them were placed at $8 \mathrm{~cm}$ intervals.

The onions, about 100 in number, used for description were chosen randomly from all of the replication. Unequal numbers of onions were taken for observation due to the fact that the plots were attacked by neck rot.

The following quantitative traits of onion bulbs were studied (Table 3-9): weight (Table 3, Fig. 2) in dag, height in $\mathrm{cm}$ (Fig. 3), diameter in $\mathrm{cm}$ (Fig. 4), shape index (ratio of the height to width), collar thickness in $\mathrm{cm}$, dry skin thickness in $\mu$, firmness (on the basis of readings from $0-100$ of a Type No. 02 durometer from The Shore Instrument and MFG. Co., Inc., 90-35 Van Wyck Expressway, Jamaica, N.Y. 11435, U.S.A.) and root disc diameter in $\mathrm{cm}$ (Fig. 1).

In addition, the UPOV (International Union for The Protection of New Varieties of Plants) evaluation degrees were used as the basis of grading the bulbs into accepted classes taking into account the following traits of the parental lines: shape (Fig. 1, Table 10), color (Table 12), and dry skin adhesion (Table 11$)-(1$ - weak, 2 - medium, 3 - good $)$ 
T a b le 1

Characteristics of the weather in Skierniewice during the 1977 and 1978 vegetation periods

\begin{tabular}{|c|c|c|c|c|c|c|c|c|c|c|c|c|c|c|c|c|c|c|c|c|}
\hline \multirow{4}{*}{ Month } & \multicolumn{12}{|c|}{ Temperature } & \multicolumn{8}{|c|}{ Rainfall } \\
\hline & \multicolumn{4}{|c|}{ monthly $\bar{x}$} & \multirow{2}{*}{\multicolumn{4}{|c|}{1977}} & \multirow{2}{*}{\multicolumn{4}{|c|}{1978}} & \multicolumn{6}{|c|}{ decade } & \multirow{2}{*}{\multicolumn{2}{|c|}{ month }} \\
\hline & \multicolumn{2}{|c|}{ lowest } & \multicolumn{2}{|c|}{ highest } & & & & & & & & & \multicolumn{2}{|c|}{ I } & \multicolumn{2}{|c|}{ II } & \multicolumn{2}{|c|}{ III } & & \\
\hline & 1977 & 1978 & 1977 & 1978 & date & lowest & date & highest & date & lowest & date & highest & 1977 & 1978 & 1977 & 1978 & 1977 & 1978 & 1977 & 1978 \\
\hline April & 2,5 & 1.8 & 10.8 & 11,0 & 1.04 & -3.3 & 30.04 & 27.8 & 9.04 & -3.7 & 24.04 & $4 \quad 18.5$ & 26.6 & 3.3 & 1.3 & 27,0 & 14.8 & 2.9 & 42.7 & 33.2 \\
\hline May & 11.3 & 6.8 & 17.5 & 17.2 & 27.05 & 1.1 & 2,05 & 26.8 & 12.05 & -0.2 & 31.05 & 525.1 & 28.0 & 40.4 & 45.6 & 0.3 & 14.7 & 2.1 & 88.3 & 42.8 \\
\hline June & 11.5 & 10.1 & 22.2 & 20.7 & 3.06 & 2.4 & 9.06 & 28.2 & 17.06 & 4.1 & 7.06 & 628.0 & 5.5 & 8.7 & 35.0 & 5.7 & 11.3 & 32.2 & 51.8 & 47.6 \\
\hline July & 11.7 & 11.1 & 21.5 & 21.1 & 16.07 & 8.1 & 25.07 & 28.6 & 18.07 & 7.2 & 31.07 & $\begin{array}{ll}7 & 28.2\end{array}$ & 77.3 & 44.5 & 10.2 & 2.0 & 44.3 & 14.2 & 131.8 & 60.7 \\
\hline August & 12.0 & 11.9 & 20.7 & 20.9 & 18.08 & 6.5 & 31.08 & 26.4 & 28.08 & 7.0 & 2.08 & 28.7 & 34.5 & 26.7 & 85.2 & 61.7 & 45.0 & 7.3 & 164.7 & 95.7 \\
\hline
\end{tabular}


Table 2

Pedigree of parents, $F_{1}, F_{2}$ and no. of bulbs

\begin{tabular}{|c|c|c|c|c|c|c|c|c|c|c|}
\hline \multirow{2}{*}{ No. } & \multirow{2}{*}{ Hybrid formula } & \multirow{2}{*}{$\begin{array}{l}\text { Maternal line } \\
\text { male sterile A }\end{array}$} & \multirow{2}{*}{ Paternal line $\mathrm{C}$} & \multicolumn{3}{|c|}{1977} & \multirow[b]{2}{*}{$\mathrm{F}_{2}$} & \multicolumn{3}{|c|}{1978} \\
\hline & & & & line A & line $C$ & $F_{1}$ & & line $A$ & line $C$ & $F_{1}$ \\
\hline 1. & A-6 $\times$ C-177a & Wolska ZW-H & 'Rawska' & 100 & 100 & 88 & 100 & - & - & - \\
\hline 2. & A-16 $\times$ C-177a & Wolska ZW-H & 'Rawska' & 100 & 100 & 80 & 100 & 65 & 31 & 96 \\
\hline 3. & A-54 $\times$ C- $-177 a$ & Wolska ZW-H & 'Rawska' & 100 & 100 & 91 & 100 & 100 & 31 & 100 \\
\hline 4. & A- $58 \times C-177 a$ & Wolska ZW-H & 'Rawska' & 79 & 100 & 72 & - & - & - & - \\
\hline 5. & A- $125 \times$ C- $-177 a$ & Wolska ZW-H & 'Rawska' & 85 & 100 & 100 & 100 & 97 & 31 & 19 \\
\hline 6. & A-19 $\times$ C- $-177 a$ & SW 34 A2 Dako & 'Rawska' & 67 & 100 & 61 & - & - & - & - \\
\hline 7. & A-181 $\times$ C- $-177 a$ & SW 34 A2 Dako & 'Rawska' & 100 & 100 & 100 & - & 100 & 31 & 100 \\
\hline 8. & A- $63 \times C-177 b$ & (Ia467-8 W101B) Rawska & 'Rawska' & 100 & 90 & 100 & 100 & - & - & 一 \\
\hline 9. & A-16 $\times$ C-141 & Wolska ZW-H & 'Rawska' & 100 & 100 & 90 & 90 & 一 & - & 一 \\
\hline 10. & A.58 $\times$ C-141 & Wolska ZW-H & 'Rawska' & 79 & 100 & 100 & - & 100 & 67 & 67 \\
\hline 11. & A-12 $\times$ C-141 & Wolska ZW-H & 'Rawska' & - & - & - & - & 56 & 67 & 92 \\
\hline 12. & A- $125 \times$ C-181 & Wolska ZW-H & 'Kutnowska' & 85 & 100 & 100 & 100 & 97 & 100 & 100 \\
\hline 13. & $A-181 \times C-181$ & SW 34 A2 Dako & 'Kutnowska' & 一 & - & - & - & 100 & 100 & 100 \\
\hline 14. & A-16 $\times$ C-16 & Wolska ZW-H & 'Wolska H & 100 & 100 & 100 & 100 & 65 & 100 & 100 \\
\hline 15. & A-6 $\times$ C- 6 & Wolska ZW-H & 'Rawska' & 100 & 100 & 100 & 100 & - & - & - \\
\hline 16. & A-125 $\times$ C-199 & Wolska ZW-H & 'Rawska' & 85 & 100 & 80 & 100 & 一 & - & - \\
\hline 17. & $\mathrm{~A}-19 \times \mathrm{C}-19$ & SW 34 A2 Dako & 'Kutnowska' & 67 & 100 & 100 & 100 & 一 & - & - \\
\hline 18. & A-16 $\times$ C-9 & Wolska ZW-H & 'Wolska H' & - & - & - & - & 65 & 67 & 100 \\
\hline 19. & A-101 $\times$ C-9 & SW 34 A2 Dako & 'Wolska H' & - & - & - & - & 100 & 67 & 26 \\
\hline 20. & A-181 $\times$ C-9 & SW 34 A2 Dako & 'Wolska H' & - & - & - & - & 100 & 67 & 98 \\
\hline
\end{tabular}


Table 3

Standard deviation $(Q)$, variability coefficient $(V \%)$ and heritability $\left(h^{2}\right)$ of onion bulb weight of parental lines and $F_{1}$ and $F_{2}$ hybrids

\begin{tabular}{|c|c|c|c|c|c|c|c|c|c|c|c|c|c|c|c|c|}
\hline & \multirow{3}{*}{ Hybrid formula } & \multicolumn{5}{|c|}{1977} & & & & & \multicolumn{6}{|c|}{1978} \\
\hline & & \multicolumn{2}{|c|}{$\begin{array}{c}\text { Maternal line } \\
\text { A }\end{array}$} & \multicolumn{2}{|c|}{$\begin{array}{c}\text { Paternal line } \\
\text { C }\end{array}$} & \multicolumn{3}{|c|}{$F_{1}$} & \multicolumn{2}{|l|}{$\mathrm{F}_{2}$} & \multicolumn{4}{|c|}{$\begin{array}{cc}\text { Maternal line } & \text { Paternal line } \\
\text { A } & \text { C } \\
\end{array}$} & \multicolumn{2}{|c|}{$F_{1}$} \\
\hline & & $\sigma$ & $\mathrm{V} \%$ & $\sigma$ & $\mathrm{V} \%$ & $\sigma$ & $\mathrm{V} \%$ & $\sigma$ & V\% & $\mathrm{h}^{2}$ & $\sigma$ & $V \sigma \%$ & & V\% & $\sigma$ & $\mathrm{V} \%$ \\
\hline 1. & A-6 $\times$ C-177a & 2.35 & 19.6 & 1.66 & 17.5 & 2.93 & 25.2 & 2.83 & 37.0 & 0.51 & - & 一 & - & - & - & - \\
\hline 2. & A-16 $\times$ C-177a & 2.67 & 24.3 & 1.66 & 17.5 & 4.10 & 26.1 & 3.48 & 41.1 & 0.63 & 3.92 & 30.2 & 2.14 & 32.2 & 3.38 & 28.1 \\
\hline 3. & A-54 $\times$ C-177a & 3.21 & 19.2 & 1.66 & 17.5 & 3.38 & 24.0 & 2.57 & 42.8 & 0.20 & 3.14 & 33.0 & 2.14 & 32.2 & 3.80 & 32.2 \\
\hline 4. & A-58 $\times$ C-177a & 4.16 & 31.1 & 1.66 & 17.5 & 4.88 & 36.7 & - & - & 一 & - & - & - & - & - & - \\
\hline 5. & A-125 $\times$ C-177a & 3.38 & 28.0 & 1.66 & 17.5 & 3.44 & 23.8 & 4.14 & 48.2 & 0.67 & 3.21 & 42.8 & 2.14 & 32.2 & 3.83 & 49.0 \\
\hline 6. & A-19 $\times$ C-177a & 3.93 & 33.0 & 1.66 & 17.5 & 4.25 & 30.8 & - & - & - & - & - & 一 & $\cdots$ & - & - \\
\hline 7. & A-181 $\times$ C-177a & 6.68 & 37.0 & 1.66 & 17.5 & 4.33 & 30.8 & - & - & - & 3.91 & 35.2 & 2.14 & 32.2 & 2.95 & 33.6 \\
\hline 8. & A-63 $\times C-177 b$ & 1.95 & 21.2 & 3.72 & 33.0 & 3.40 & 26.0 & 5.50 & 51.8 & 0,76 & - & - & 一 & - & - & - \\
\hline 9. & A-16 $\times$ C-141 & 2.67 & 24.3 & 2.23 & 23.8 & 3.99 & 29.1 & 4.60 & 36.8 & 0,72 & - & - & - & - & - & - \\
\hline 10. & A-58 $\times$ C-141 & 4.16 & 31.1 & 2.23 & 23.8 & 4.88 & 36.7 & - & - & - & 3.53 & 33.9 & 1.80 & 31.0 & 3.11 & 38.8 \\
\hline 11. & A-12 $\times$ C-141 & - & - & - & 一 & - & - & $一$ & - & - & 3.16 & 33.6 & 1.80 & 31.0 & 2.80 & 21.9 \\
\hline 12. & A-125 $\times$ C-181 & 3.38 & 28.0 & 4.35 & 29.0 & 4.36 & 25.8 & 3.37 & 41.7 & $-0,30$ & 3.21 & 42.8 & 5.16 & 56.8 & 5.29 & 37.2 \\
\hline 13. & A-181 $\times$ C-181 & - & - & 一 & - & - & - & - & - & - & 3.91 & 35.2 & 5.16 & 56.8 & 5.24 & 40.4 \\
\hline 14. & A-16 $\times$ C-16 & 2.67 & 24.3 & 5.35 & 34.5 & 5.51 & 35.2 & 4.13 & 30.2 & 0.16 & 3.92 & 30.2 & 5.21 & 45.8 & 5.39 & 56.5 \\
\hline 15. & A-6 $\times$ C- 6 & 2.35 & 19.6 & 1.71 & 22.7 & 4.10 & 32.0 & 3.63 & 32.5 & 0.70 & - & - & - & - & - & - \\
\hline 16. & A $125 \times$ C 199 & 3.38 & 28.0 & 3.33 & 32.7 & 3.61 & 25.4 & 3.56 & 31.9 & 0.11 & - & - & - & - & - & - \\
\hline 17. & A-19 $\times$ C-19 & 3.93 & 33.0 & 2.45 & 22.2 & 6.51 & 37.7 & 5.07 & 36.8 & 0.63 & $\cdots$ & - & - & - & - & - \\
\hline 18. & A-16 $\times$ C-9 & - & - & - & - & - & 一 & - & - & - & 3.92 & 30.2 & 2.36 & 36.7 & 4.14 & 34.7 \\
\hline 19. & A-101 $\times$ C-9 & 一 & - & - & - & - & - & - & $二$ & - & 4.24 & 37.8 & 2.36 & 36.7 & 6.15 & 58.1 \\
\hline 20. & A-181 $\times$ C-9 & - & - & - & - & - & 一 & 一 & - & - & 3.91 & 33.2 & 2.36 & 36.7 & 5.22 & 33.8 \\
\hline
\end{tabular}


Table 4

Standard deviation $(\sigma)$, variability coefficient $(V \%)$ and heritability $\left(h^{2}\right)$ of onion bulb height $(\mathrm{cm})$ of parental forms and $F_{1}$ and $F_{2}$ hybrids

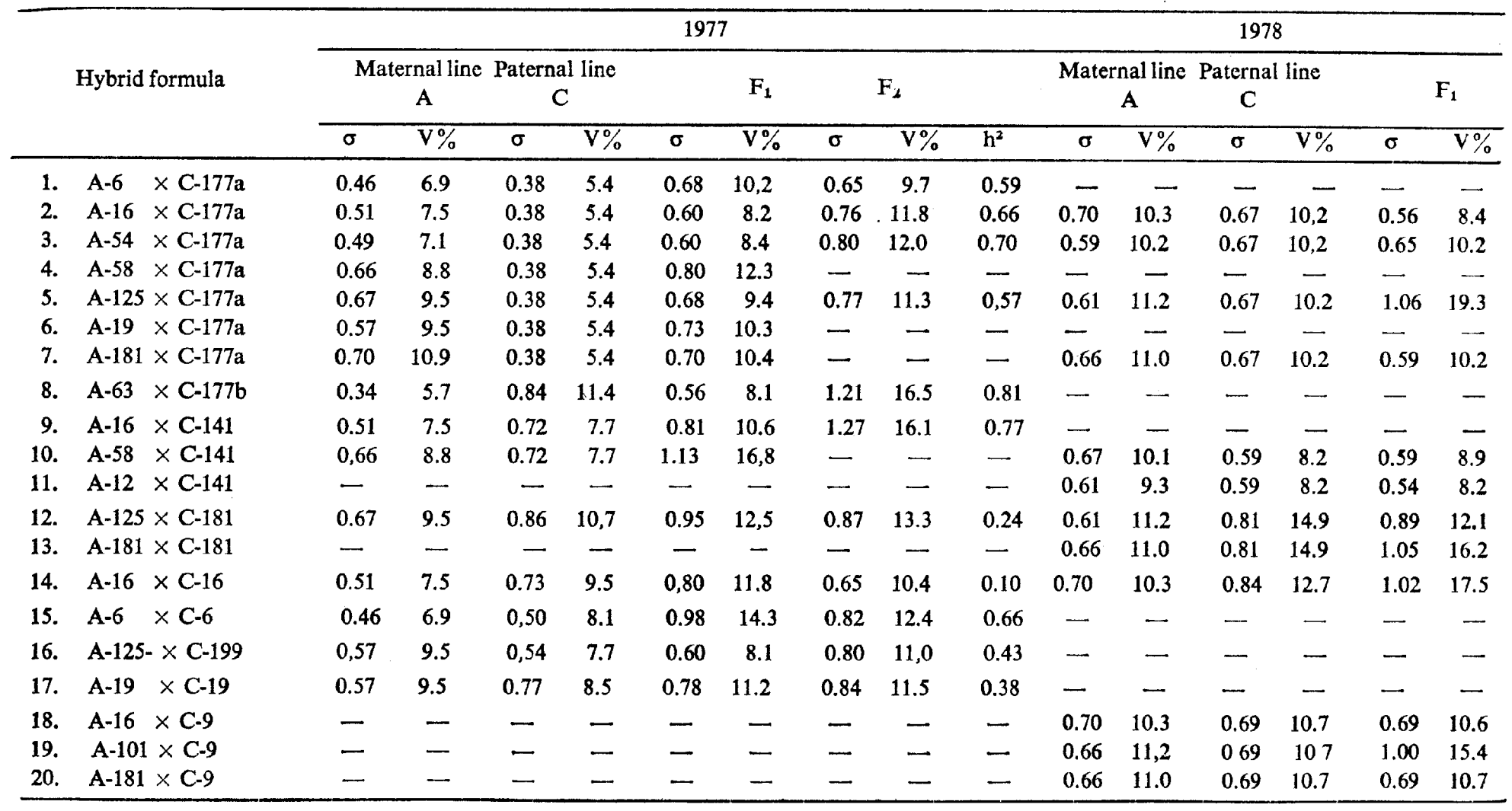


Tab le 5

Standard deviation $(\sigma)$, variability coefficient $(V \%)$ and heritability $\left(h^{2}\right)$ of bulb diameter $(\mathrm{cm})$ of parental lines and $F_{1}$ and $F_{2}$ hybrids

\begin{tabular}{|c|c|c|c|c|c|c|c|c|c|c|c|c|c|c|c|c|c|}
\hline & \multirow{3}{*}{\multicolumn{2}{|c|}{ Hybrid formula }} & \multicolumn{9}{|c|}{1977} & \multicolumn{6}{|c|}{1978} \\
\hline & & & \multicolumn{4}{|c|}{$\begin{array}{cc}\text { Maternal line } & \text { Paternal line } \\
\text { A } & \text { C }\end{array}$} & \multicolumn{3}{|c|}{$F_{1}$} & \multicolumn{2}{|l|}{$F_{2}$} & \multicolumn{4}{|c|}{$\begin{array}{cc}\text { Maternal line } & \text { Paternal line } \\
\text { A } & \text { C }\end{array}$} & \multicolumn{2}{|c|}{$F_{1}$} \\
\hline & & & $\sigma$ & $\mathrm{V} \%$ & $\sigma$ & $\mathrm{V} \%$ & $\sigma$ & $V \%$ & $\sigma$ & $\mathrm{V} \%$ & $h^{2}$ & $\sigma$ & $\mathrm{V} \%$ & $\sigma$ & $V \%$ & $\sigma$ & $\mathrm{V} \%$ \\
\hline 1. & A-6 & $\times \mathrm{C}-177 \mathrm{a}$ & 0.59 & 9.2 & 0.41 & 7.5 & 0.62 & 9.8 & 0.72 & 14.3 & 0.54 & 一 & 一 & $\rightarrow$ & - & 一 & 一 \\
\hline 2. & A-16 & $\times \mathrm{C}-177 \mathrm{a}$ & 0.55 & 8.9 & 0.41 & 7.5 & 0.77 & 11.1 & 0.86 & 15.8 & 0.70 & 0.77 & 11.7 & 0.59 & 12.6 & 0,68 & 10,8 \\
\hline 3. & A-54 & $\times \mathrm{C}-177 \mathrm{a}$ & 0.53 & 7.1 & 0.41 & 7,5 & 0.63 & 9.4 & 0.77 & 16.5 & 0.64 & 0.76 & 12.6 & 0.59 & 12.6 & 0.75 & 11.8 \\
\hline 4. & A-58 & $\times \mathrm{C}-177 \mathrm{a}$ & 0.76 & 11.4 & 0,41 & 7.5 & 0,84 & 13.8 & - & - & - & - & - & - & - & - & 一 \\
\hline 5. & A-125 & $\times \mathrm{C}-177 \mathrm{a}$ & 0.68 & 10.7 & 0.41 & 7.5 & 0.60 & 9.0 & 0.96 & 18.1 & 0.69 & 0.86 & 16.0 & 0.59 & 12.6 & 1.02 & 20.2 \\
\hline 6. & A-19 & $\times \mathrm{C}-177 \mathrm{a}$ & 0.94 & 13.8 & 0.41 & 7.5 & 0.74 & 11.2 & - & - & - & - & - & - & - & - & - \\
\hline 7. & A-181 & $\times C-177 a$ & 1.23 & 15.6 & 0.41 & 7.5 & 0.81 & 11.7 & - & - & - & 0.87 & 13.8 & 0.59 & 12.6 & 0.69 & 12.0 \\
\hline 8. & A-63 & $\times C-177 b$ & 0.48 & 8.3 & 0.72 & 12.2 & 0,61 & 9.8 & 1.13 & 19.5 & 0.73 & - & - & - & - & - & - \\
\hline 9. & A-16 & $\times \mathrm{C}-141$ & 8.55 & 8.9 & 0.42 & 8.9 & 0.68 & 10.5 & 0.92 & 15.1 & 0.73 & - & $一$ & - & $\ldots$ & - & - \\
\hline 10 & A-58 & × C-141 & 0.76 & 11.4 & 0.42 & 8.9 & 0.77 & 11.9 & - & & - & 0.85 & 14.0 & 0.47 & 11.1 & 0.78 & 14.7 \\
\hline 11. & A-12 & $\times$ C-141 & - & 一 & - & - & - & - & - & $\cdots$ & $\cdots$ & 0.70 & 12.7 & 0.47 & 11.1 & 0.58 & 9.0 \\
\hline 12. & A-125 & X C-181 & 0.68 & 10.7 & 0.72 & 11.0 & 0.72 & 10.4 & 0.79 & 14.8 & 0.21 & 0.86 & 16.0 & 1.20 & 20.8 & 1.01 & 15.7 \\
\hline 13. & A-181 & $\times \mathrm{C}-181$ & - & - & 一 & - & - & - & - & - & - & 0.87 & 13.8 & 1.20 & 20.8 & 1.10 & 16.8 \\
\hline 14. & A-16 & $\times \mathrm{C}-16$ & 0,55 & 8.9 & 0.96 & 14.0 & 0.94 & 13.2 & 0.76 & 10.8 & 0.09 & 0.77 & 11.7 & 1.08 & 17.9 & 1.08 & 18.3 \\
\hline 15. & A-6 & $\times \mathrm{C}-6$ & 0.59 & 9.2 & 0.46 & 8.4 & 0.81 & 12.4 & 0.79 & 12.8 & 0.56 & - & - & - & - & - & - \\
\hline 16. & A-125 & × C-199 & 0.68 & 10.7 & 0.66 & 11.7 & 0.67 & 10.1 & 0.72 & 12.5 & 0.13 & - & - & - & - & 一 & - \\
\hline 17. & A-19 & $\times$ C-19 & 0.94 & 13.8 & 0.48 & 8.7 & 1,07 & 14.5 & 1.00 & 14.8 & 0.55 & 一 & - & - & - & 一 & 一 \\
\hline 18. & A-16 & $\times$ C -9 & - & - & - & - & - & - & - & 一 & - & 0.77 & 11.7 & 0.65 & 14.2 & 0.87 & 13.6 \\
\hline 19. & A-101 & $\times$ C -9 & - & - & - & 一 & 一 & - & - & - & - & 0.93 & 14.8 & 0,65 & 14.2 & 1.31 & 22.0 \\
\hline 20. & A-181 & $\times \mathrm{C}-9$ & 一 & - & - & - & - & 一 & 一 & - & 一 & 0.87 & 13.8 & 065 & 14.2 & 0.91 & 12.8 \\
\hline
\end{tabular}




\section{T a b le 6}

Standard deviation $(\sigma)$, variability coefficient $(V \%)$ and heritability $\left(h_{2}\right)$ of collar thickness $(\mathrm{cm})$ of parental lines and $F_{1}$ and $F_{2}$ hybrids

\begin{tabular}{|c|c|c|c|c|c|c|c|c|c|c|c|c|c|c|c|c|c|}
\hline \multirow{3}{*}{ 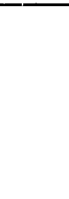 } & \multirow{3}{*}{\multicolumn{2}{|c|}{ Hybrid formula }} & \multicolumn{9}{|c|}{1977} & \multicolumn{6}{|c|}{1978} \\
\hline & & & \multicolumn{4}{|c|}{$\begin{array}{cc}\text { Maternal line } & \text { Paternal line } \\
\text { A } & \text { C }\end{array}$} & \multicolumn{3}{|c|}{$F_{1}$} & \multicolumn{2}{|l|}{$F_{2}$} & \multicolumn{4}{|c|}{$\begin{array}{cc}\text { Maternal line Paternal line } \\
\text { A }\end{array}$} & \multicolumn{2}{|c|}{$F_{1}$} \\
\hline & & & $\sigma$ & $\mathrm{V \%}$ & $\sigma$ & $V \%$ & $\sigma$ & $A \%$ & $\sigma$ & $V \%$ & $h^{2}$ & $\sigma$ & $V \%$ & $\sigma$ & $V \%$ & $\sigma$ & $V \%$ \\
\hline 1. & A- 6 & $\times C-177 a$ & 0.24 & 13.9 & 0.16 & 9.0 & 0.21 & 12.5 & 0.20 & 16.9 & 0.06 & - & - & - & $\cdots$ & - & - \\
\hline 2. & A-16 & $\times C-177 a$ & 0.21 & 12.2 & 0.16 & 9.0 & .0 .24 & 13.2 & 0.22 & 17.0 & 0.32 & 0.23 & 15.3 & 0.21 & 13.6 & 0.16 & 10.7 \\
\hline 3. & A-54 & $\times \mathrm{C}-177 \mathrm{a}$ & 0.20 & 10.4 & 0.16 & 9.0 & 0.20 & 10.7 & 0.17 & 15.3 & -0.12 & 0.19 & 13.4 & 0.21 & 13.6 & 0.18 & 12.8 \\
\hline 4. & A-58 & $\times C-177 a$ & 0.19 & 10.7 & 016 & 9.0 & 0.22 & 14.0 & - & - & $\ldots$ & - & - & - & - & - & - \\
\hline 5. & A-125 & $\times C-177 a$ & 0.26 & 14.5 & 0.16 & 9.0 & 0.27 & 13.7 & 0.26 & 19.6 & 0.38 & 0.24 & 19.6 & 0.21 & 13.6 & 0.33 & 25.9 \\
\hline 6. & A-19 & $\times \mathrm{C}-177 \mathrm{a}$ & 0.20 & 13.6 & 0.16 & 9.0 & 0.30 & 16.5 & - & - & - & $\ldots$ & - & - & - & $-\cdots$ & -- \\
\hline 7. & A-181 & $\times \mathrm{C}-177 \mathrm{a}$ & 0.35 & 22.2 & 0.16 & 9.0 & 0.26 & 15.5 & - & - & - & 0.26 & 17.8 & 0.21 & 13.6 & 0.23 & 16.1 \\
\hline 8. & A-63 & $\times \mathrm{C}-177 \mathrm{~b}$ & 0.21 & 10.9 & 0.31 & 16.6 & 0.20 & 10.2 & 0.33 & 22.7 & 0.42 & - & - & -- & - & - & $\cdots$ \\
\hline 9. & A-16 & $\times \mathrm{C}-141$ & 0.21 & 12.2 & 0.15 & 8.7 & 0.25 & 14.1 & 0.20 & 14.2 & 0.22 & - & - & - & - & - & - \\
\hline 10. & A-58 & $\times$ C-141 & 0.19 & 10.7 & 0.15 & 8.7 & 0.29 & 19.5 & - & - & - & 0.24 & 16.6 & 0.15 & 12.2 & 0.18 & 13.9 \\
\hline 11. & A-12 & $\times \mathrm{C}-141$ & - & - & $\cdots$ & - & - & - & - & - & - & 0.22 & 14.2 & 0.15 & 12.2 & 0.19 & 12.7 \\
\hline 12. & A-125 & $\times$ C-181 & 0.26 & 14.5 & 0.37 & 19.8 & 0.26 & 18.5 & 0.19 & 15.8 & -1.78 & 0.24 & 19.6 & 0.31 & 23.5 & 0.24 & 15.3 \\
\hline 13. & A-181 & $\times \mathrm{C}-181$ & - & - & - & - & - & - & - & - & - & 0.26 & 17.8 & 0.31 & 23.5 & 0.30 & 20.3 \\
\hline 14. & A-16 & $\times \mathrm{C}-16$ & 0.21 & 12.2 & 0.30 & 16.8 & 0.29 & 17.8 & 0.13 & 10.8 & -2.66 & 0.23 & 15.3 & 0.34 & 20.4 & 0.27 & 20.2 \\
\hline 15. & A-6 & $\times$ C-6 & 0.24 & 13.9 & 0.16 & 9.9 & 0,34 & 18.2 & 0.20 & 14.9 & 0.08 & - & - & - & - & - & - \\
\hline 16. & A-125 & $\times$ C-199 & 0.26 & 14.5 & 0.28 & 15.4 & 0.23 & 12.1 & $0.2 \mathrm{l}$ & 15.2 & -0.56 & - & - & - & - & - & - \\
\hline 17. & A-19 & $\times$ C-19 & 0.20 & 13.6 & 0.26 & 13.7 & 0.34 & 18.6 & 022 & 14.6 & 0.02 & $\cdot-$ & $\ldots$ & - & - & - & - \\
\hline 18. & A-16 & $\times$ C-9 & - & - & - & - & - & - & - & - & - & 0.23 & 15.3 & 0.16 & 12.0 & 0.23 & 15.3 \\
\hline 19. & A-101 & $\times$ C -9 & - & - & - & - & - & - & - & - & $\cdots$ & 0.29 & 20.0 & 0.16 & 12.0 & 0.39 & 26.1 \\
\hline 20. & A-181 & $\times$ C -9 & - & - & - & - & - & - & $\ldots$ & - & - & 0.26 & 17.8 & 0.16 & 12.0 & 0.22 & 15.2 \\
\hline
\end{tabular}


Table 7

Standard deviation $(\sigma)$, variability coefficient $(V \%)$ and heritability $\left(h^{2}\right)$ of dry skin thickness $(\mu)$ of parental lines and $F_{1}$ and $F_{2}$ hybrids

\begin{tabular}{|c|c|c|c|c|c|c|c|c|c|c|c|c|c|c|c|c|}
\hline & \multirow{3}{*}{ Hybrid formula } & \multicolumn{9}{|c|}{1977} & \multicolumn{6}{|c|}{1978} \\
\hline & & \multicolumn{4}{|c|}{$\begin{array}{cc}\text { Maternal line } & \text { Paternal line } \\
\text { A } & \text { C }\end{array}$} & \multicolumn{2}{|c|}{$F_{1}$} & \multicolumn{3}{|c|}{$F_{2}$} & \multicolumn{4}{|c|}{$\begin{array}{cc}\text { Maternal line } & \text { Paternal line } \\
\text { A } & \text { C }\end{array}$} & \multicolumn{2}{|c|}{$F_{t}$} \\
\hline & & $\sigma$ & $V \%$ & $\sigma$ & $V \%$ & $\sigma$ & $V \%$ & $\sigma$ & V\% & $h^{2}$ & $\sigma$ & $V \%$ & $\sigma$ & $V \%$ & $\sigma$ & $\mathrm{V} \%$ \\
\hline 1. & $\times C-177 a$ & 0.07 & 29.9 & 0.20 & 31.2 & 0.13 & 28.9 & 0.14 & 31.1 & 0.34 & - & - & - & - & - & - \\
\hline 2. & A-16 $\times$ C-177a & 0.08 & 28.6 & 0.20 & 31.2 & 0.13 & 31.7 & 0.13 & 35.1 & 0.08 & 0.10 & 30.8 & 0.15 & 25.2 & 0.10 & 27.4 \\
\hline 3. & A-54 $\times$ C-177a & 0.09 & 28.8 & 0.20 & 31.2 & 0.13 & 32.5 & 0.15 & 31.0 & 0.28 & 0.08 & 35.4 & 0.15 & 25.2 & 0.10 & 30.8 \\
\hline 4. & A-58 $\times$ C-177a & 0.08 & 26.4 & 0.20 & 31.2 & 0.10 & 26.2 & - & - & - & - & 一 & - & - & - & - \\
\hline 5. & A-125 $\times$ C $-177 a$ & 0.08 & 28.5 & 0.20 & 31.2 & 0.10 & 30.0 & 0.13 & 32.0 & 0.08 & 0.13 & 41.6 & 0.15 & 25.2 & 0.10 & 24.5 \\
\hline 6. & A-19 $\times C-177 a$ & 0.14 & 29.0 & 0.20 & 31.2 & 0.21 & 29.9 & - & - & - & - & 一 & - & - & - & - \\
\hline 7. & A-181 $\times C-177 a$ & 0.12 & 31.1 & 0.20 & 31.2 & 0.17 & 29.3 & - & - & - & 0.14 & 33.5 & 0.15 & 25.2 & 0.17 & 27.4 \\
\hline 8. & $A-63 \times C-177 b$ & 0.09 & 22.7 & 0.18 & 35.6 & 0.11 & 23.8 & 0.13 & 30.0 & 0.11 & - & - & - & - & - & - \\
\hline 9. & A-16 $\times C-141$ & 0.08 & 28.6 & 0.12 & 24.3 & 0.09 & 28.7 & 0.12 & 26.2 & 0.32 & - & - & - & - & - & - \\
\hline 10. & $A-58 \times C-141$ & 0.08 & 26.4 & 0.12 & 24.3 & 0.11 & 27.5 & 一 & - & - & 0.09 & 38.1 & 0.16 & 27.8 & 0.13 & 36.9 \\
\hline 11. & $\mathrm{~A}-12 \times \mathrm{C}-141$ & - & - & - & - & - & - & - & 一 & - & 0.16 & 28.3 & 0.16 & 27.8 & 0.11 & 30.2 \\
\hline 12. & A-125 $\times$ C-181 & 0.08 & 28.5 & 0.09 & 23.4 & 0.15 & 30.4 & 0.14 & 32.5 & 0.62 & 0.13 & 41.6 & 0.16 & 35.0 & 0.14 & 37.6 \\
\hline 13. & $\mathrm{~A}-181 \times \mathrm{C}-181$ & 一 & - & - & 一 & - & - & - & - & - & 0.14 & 33.5 & 0.16 & 35.0 & 0.12 & 29.8 \\
\hline 14. & $A-16 \times C-16$ & 0.08 & 28.6 & 0.21 & 36.3 & 0.09 & 32.5 & 0.13 & 33.0 & 0.01 & 0.10 & 30.8 & 0.15 & 30.7 & 0.13 & 36.3 \\
\hline 15. & A- $6 \times$ C -6 & 0.07 & $29.9 \ldots$ & 0.11 & 30.6 & 0.08 & 26.8 & 0.10 & 24.4 & 0.14 & 一 & - & 一 & 一 & - & - \\
\hline 16. & A-125 $\times$ C-199 & 0.08 & 28.5 & 0.28 & 39.9 & 0.12 & 29.8 & 0.12 & 27.1 & -0.67 & $\cdots$ & - & 一 & 一 & 一 & - \\
\hline 17. & $\mathrm{~A}-19 \times \mathrm{C}-19$ & 0.14 & 29.0 & 0.11 & 25.5 & 0.09 & 26.9 & 0.13 & 32.8 & 0.11 & - & - & - & - & - & 一 \\
\hline 18. & A-16 $\times$ C -9 & - & - & - & - & - & - & - & - & - & 0.10 & 30.8 & 0.18 & 34.5 & 0.10 & 33.2 \\
\hline 19. & A-101 $\times$ C-9 & - & - & - & - & 一 & - & 一 & - & - & 0.11 & 25.3 & 0.18 & 34.5 & 0.17 & 47.0 \\
\hline 20. & A-181 $\times$ C-9 & - & - & - & - & - & - & - & - & - & 0.14 & 33.5 & 0.18 & 34.5 & 0.11 & 30.9 \\
\hline
\end{tabular}


Table 8

Standard deviation $(\sigma)$, variability coefficient $(V \%)$ and heritability $\left(h^{2}\right)$ of bulb firmness of parental lines and $F_{1}$ and $F_{2}$ hybrids

\begin{tabular}{|c|c|c|c|c|c|c|c|c|c|c|c|c|c|c|c|c|}
\hline & \multirow{3}{*}{ Hybrid formula } & \multicolumn{9}{|c|}{1977} & \multicolumn{6}{|c|}{1978} \\
\hline & & \multicolumn{4}{|c|}{$\begin{array}{cc}\text { Maternal line } & \text { Paternal line } \\
\text { A } & \text { C }\end{array}$} & \multicolumn{3}{|c|}{$\mathrm{F}_{1}$} & \multicolumn{2}{|l|}{$\mathrm{F}_{2}$} & \multicolumn{4}{|c|}{$\begin{array}{cc}\text { Maternal line } & \text { Paternal line } \\
\text { A } & \text { C } \\
\end{array}$} & \multicolumn{2}{|c|}{$F_{1}$} \\
\hline & & $\sigma$ & $\mathrm{V} \%$ & $\sigma$ & $\mathrm{V} \%$ & $\sigma$ & $\mathrm{V} \%$ & $\sigma$ & VW & $h^{2}$ & $\sigma$ & VW & $\sigma$ & VW & $\sigma$ & VW \\
\hline 1. & A-6 $\times$ C-177a & 5.47 & 7.0 & 2.79 & 3.3 & 3.40 & 4.1 & 4.77 & 5.9 & 0.33 & 一 & - & 一 & - & 一 & - \\
\hline 2. & A-16 $\times$ C-177a & 4.38 & 5.5 & 2.79 & 3.3 & 2.69 & 3.2 & 4.32 & 5.3 & 0.34 & 5.55 & 7.2 & 4.62 & 5.7 & 5.25 & 6.5 \\
\hline 3. & A-54 $\times$ C-177a & 5.10 & 6.5 & 2.79 & 3.3 & 4.00 & 4.8 & 4.16 & 5.3 & 0.18 & 5.06 & 6.6 & 4.62 & 5.7 & 4.22 & 5.3 \\
\hline 4. & A- $58 \times C-177 a$ & 5.66 & 7.4 & 2.79 & 3.3 & 6.43 & 8.1 & - & - & - & - & - & - & - & - & - \\
\hline 5. & A-125 $\times$ C- $-177 a$ & 3.77 & 4.7 & 2.79 & 3.3 & 3.68 & 4.4 & 4.53 & 5.6 & 0.49 & 4.74 & 6.0 & 4.62 & 5.7 & 12.72 & 18.7 \\
\hline 6. & A-19 $\times$ C-177a & 3.69 & 4.5 & 2.79 & 3.3 & 3.55 & 4.2 & - & 一 & - & - & - & - & - & - & 一 \\
\hline 7. & A-181 $\times$ C-177a & 5.40 & 6.9 & 2.79 & 3.3 & 3.87 & 4.7 & 一 & - & $\cdots$ & 3.66 & 4.4 & 4.62 & 5.7 & 10.63 & 13.8 \\
\hline 8. & A- $63 \times C-177 b$ & 3.60 & 4.3 & 3.89 & 4.7 & 3.76 & 4.6 & 4.78 & 6.1 & 0.39 & - & 一 & - & - & - & - \\
\hline 9. & A-16 $\times$ C-141 & 4.38 & 5.5 & 2.75 & 3.4 & 5.24 & 6.6 & 3.78 & 4.6 & 0.16 & - & - & - & 一 & - & - \\
\hline 10. & A-58 $\times$ C-141 & 5.66 & 7.4 & 2.75 & 3.4 & 6.77 & 8.4 & 一 & - & 一 & 4.88 & 6.5 & 4.11 & 5.2 & 3.75 & 4.7 \\
\hline 11. & A-12 $\times$ C-141 & - & 一 & - & - & - & - & 一 & - & - & 5.68 & 7.1 & 4.11 & 5.2 & 5.03 & 6.5 \\
\hline 12. & A-125 $\times$ C-181 & 3.77 & 4.7 & 4,81 & 5.9 & 5.86 & 7.0 & 5.35 & 6.7 & 0.37 & 4.77 & 6.0 & 6.62 & 8.7 & 3.44 & 4.2 \\
\hline 13. & A-181 $\times$ C-181 & - & - & 一 & - & - & 一 & - & - & 一 & 3.66 & 4.4 & 6.62 & 8.7 & 9.43 & 12.4 \\
\hline 14. & A-16 $\times$ C-16 & 4.38 & 5.5 & 3.65 & 4.5 & 5.02 & 6.2 & 4.55 & 5.8 & 0.23 & 5.55 & 7.2 & 5.34 & 6.8 & 6.79 & 8.9 \\
\hline 15. & A- $6 \times$ C -6 & 5.47 & 7.0 & 3.25 & 4.1 & 2.65 & 3.2 & 4.38 & 5.5 & 0.07 & - & - & - & - & 一 & - \\
\hline 16. & A-125 $\times$ C-199 & 3.77 & 4.7 & 3.41 & 4.0 & 3.35 & 4.0 & 3.38 & 4.1 & -0.13 & - & - & - & 一 & 一 & - \\
\hline 17. & $\mathrm{~A}-19 \times \mathrm{C}-19$ & 3.69 & 4.5 & 3.31 & 4.1 & 5.34 & 6.3 & 4.46 & 5.6 & 0.38 & - & 一 & - & 一 & - & 一 \\
\hline 18. & A-16 $\times$ C-9 & - & - & - & 一 & - & - & - & - & - & 5.55 & 7.2 & 3.86 & 4.8 & 3.32 & 4.2 \\
\hline 19. & A-101 $\times$ C-9 & - & - & - & $\cdots$ & - & - & $\cdots$ & $\cdots$ & $\cdots$ & 6.97 & 9.7 & 3.86 & 4.8 & 8.16 & 10.9 \\
\hline 20. & A-181 $\times$ C-9 & 一 & 一 & 一 & - & 一 & - & - & - & 一 & 3.66 & 4.4 & 3.86 & 4.8 & 3.69 & 4.8 \\
\hline
\end{tabular}


Table 9

Standard deviation $(\sigma)$ variability coefficient $(V \%)$ and heritability $\left(h^{2}\right)$ of onion disc diameter $(\mathrm{cm})$ of parental lines and $F_{1}$ and $F_{2}$ hybrids

\begin{tabular}{|c|c|c|c|c|c|c|c|c|c|c|c|c|c|c|c|c|}
\hline & \multirow{3}{*}{ Hybrid formula } & \multicolumn{9}{|c|}{1977} & \multicolumn{6}{|c|}{1978} \\
\hline & & \multicolumn{4}{|c|}{$\begin{array}{cc}\text { Maternal line } & \text { Paternal line } \\
\text { A } & \text { C }\end{array}$} & \multicolumn{3}{|c|}{$F_{1}$} & \multicolumn{2}{|l|}{$\mathrm{F}_{2}$} & \multicolumn{4}{|c|}{$\begin{array}{cc}\text { Maternal line } & \text { Paternal line } \\
\mathbf{A} & \mathrm{C} \\
\end{array}$} & \multicolumn{2}{|c|}{$F_{1}$} \\
\hline & & $\sigma$ & $\mathrm{V} \%$ & $\sigma$ & $6 \mathrm{~V} \%$ & $\sigma$ & $\mathrm{V} \%$ & $\sigma$ & $\mathrm{V} \%$ & $h^{2}$ & $\sigma$ & $\mathrm{V} \%$ & $\sigma$ & $\mathrm{V} \%$ & $\sigma$ & $V \%$ \\
\hline 1. & A-6 $\times$ C-177a & 0.15 & 10.5 & 0.14 & 11.0 & 0.17 & 13.5 & 0.18 & 15.4 & 0.32 & $\cdots$ & - & - & - & 一 & 一 \\
\hline 2. & A-16 $\times$ C-177a & 0.14 & 10.1 & 0.14 & 11.0 & 0.15 & 11.1 & 0.18 & 14.8 & 0.38 & 0.20 & 14.2 & 0.15 & 12.7 & 0.17 & 12.0 \\
\hline 3. & A-54 $\times$ C-177a & 0.15 & 10.0 & 0.14 & 11.0 & 0.14 & 10.9 & 0.17 & 15.1 & 0.26 & 0.15 & 11.3 & 0.15 & 12.7 & 0.17 & 13.4 \\
\hline 4. & A-58 $\times$ C-177a & 0.17 & 11.6 & 0.14 & 11.0 & 0.14 & 10.3 & - & - & - & - & - & - & 一 & 一 & - \\
\hline 5. & A-125 $\times$ C-177a & 0.15 & 10.7 & 0.14 & 11.0 & 0.15 & 11.1 & 0.21 & 16.9 & 0,51 & 0,16 & 13.6 & 0.15 & 12.7 & 0.16 & 14.6 \\
\hline 6. & A-19 $\times$ C-177a & 0.18 & 13.5 & 0.14 & 11.0 & 0.15 & 11.9 & - & 一 & $\cdots$ & $\cdots$ & - & - & - & - & - \\
\hline 7. & A-181 $\times$ C-177a & 0.23 & 16.2 & 0.14 & 11.0 & 0.17 & 13.5 & - & - & - & 0.17 & 12.4 & 0.15 & 12.7 & 0.14 & 12.6 \\
\hline 8. & A- $63 \times C-177 b$ & 0.11 & 8.1 & 0.15 & 11.7 & 0.15 & 11.6 & 0.24 & 18.1 & 0.70 & - & - & - & 一 & - & - \\
\hline 9. & A-16 $\times$ C-141 & 0.14 & 10.1 & 0.16 & 13.3 & 0.16 & 12.7 & 0.17 & 12.4 & 0.26 & - & - & - & 一 & $一$ & - \\
\hline 10. & A-58 $\times$ C -141 & 0.17 & 11.6 & 0.16 & 13.3 & 0.18 & 14.2 & 一 & - & - & 0.19 & 14.9 & 0.15 & 13.3 & 0.13 & 10.9 \\
\hline 11. & A-12 $\times$ C -141 & - & - & $\cdots$ & 一 & - & - & - & - & - & 0.19 & 17.2 & 0.15 & 13.3 & 0.11 & 8.7 \\
\hline 12. & A-125 $\times \mathrm{C}-181$ & 0.15 & 10.7 & 0.20 & 15.7 & 0.20 & 15.1 & 0.17 & 13.5 & -0.05 & 0.16 & 13.6 & 0.22 & 17.9 & 0.18 & 13.4 \\
\hline 13. & A-181 $\times$ C- 181 & - & - & - & - & - & - & - & - & 一 & 0.17 & 12.4 & 0.22 & 17.9 & 0.19 & 14.5 \\
\hline 14. & A-16 $\times$ C-16 & 0.14 & 10.1 & 0.17 & 12.4 & 0.16 & 11.5 & 0.12 & 8.8 & -0.63 & 0.20 & 14.2 & 0.16 & 13.9 & 0.22 & 18.1 \\
\hline 15. & A- $6 \times$ C -6 & 0.15 & 10.5 & 0.11 & 9.2 & 0.19 & 14.1 & 0.15 & 11.2 & 0.26 & - & - & 一 & - & - & 一 \\
\hline 16. & A-125 $\times$ C-199 & 0.15 & 10.7 & 0.18 & 12.8 & 0.13 & 9.7 & 0.19 & 13.9 & 0.30 & - & - & - & - & - & - \\
\hline 17. & A-19 $\times$ C- 19 & 0.18 & 13.5 & 0.14 & 10.9 & 0.19 & 15.0 & 0.13 & 10.6 & 0.41 & - & - & - & - & - & - \\
\hline 18. & A-16 $\times$ C -9 & - & - & - & - & -- & $\cdots$ & -- & - & - & 0.20 & 14.2 & 0.16 & 14.9 & 0.13 & 10.4 \\
\hline 19. & A-101 $\times$ C-9 & - & - & - & 一 & - & - & - & - & - & 0.20 & 15.0 & 0.16 & 14.9 & 0.27 & 24.7 \\
\hline 20. & A-181 $\times$ C -9 & - & 一 & - & - & - & - & - & 一 & - & 0.17 & 12.4 & 0.16 & 14.9 & 0.14 & 11.0 \\
\hline
\end{tabular}



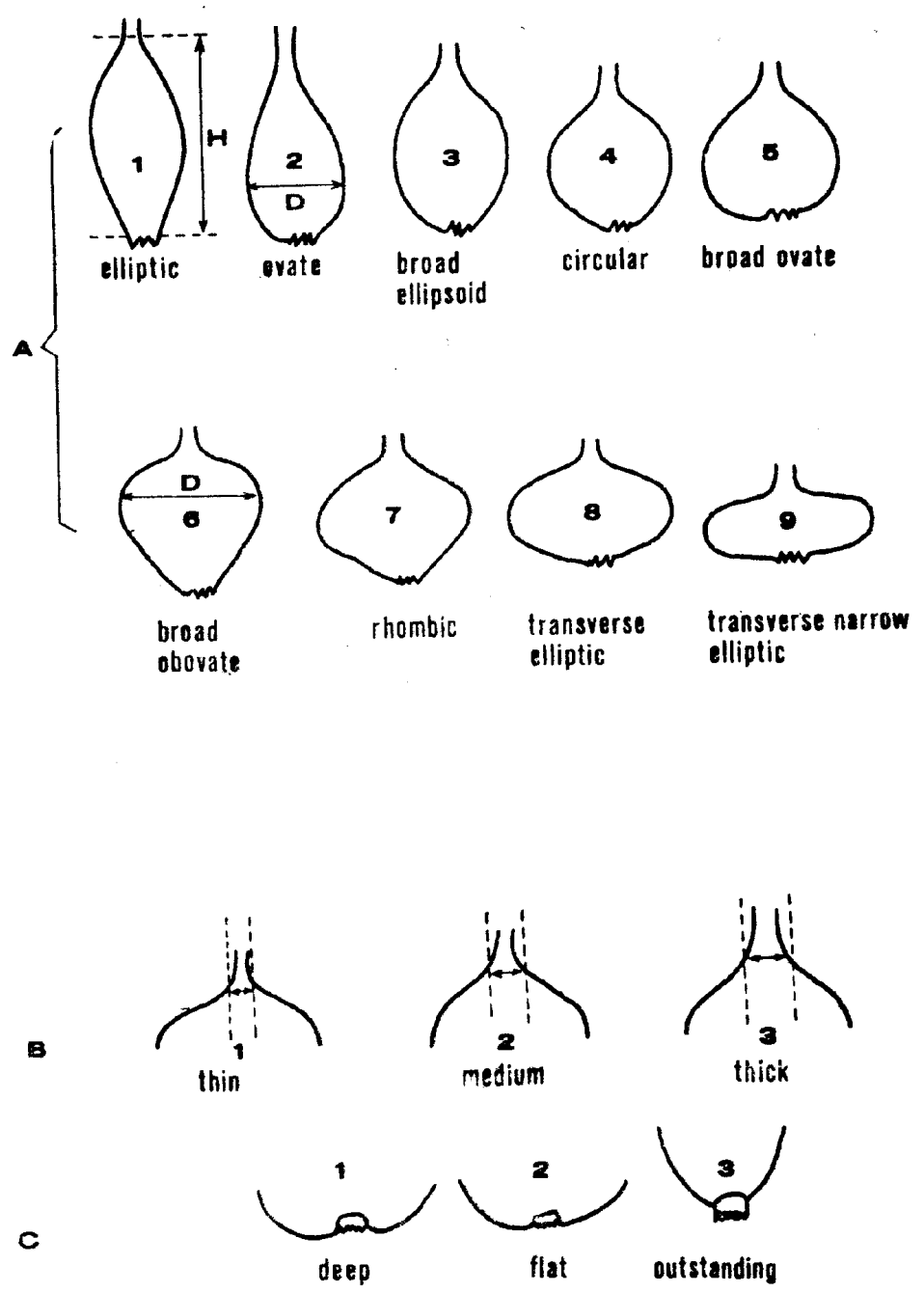

Fig. 1. Explanation of onion bulb characteristics according to UPOV Draft Guidelines. A - shape of the bulbs, B - shape of the collar, C - position of the root disc

and color of dry skin (Table 11) - (1 - greenish straw color, 2 white, 3 - light straw color, 4 - dark straw color, 5 - light brown, 6 - brown). 

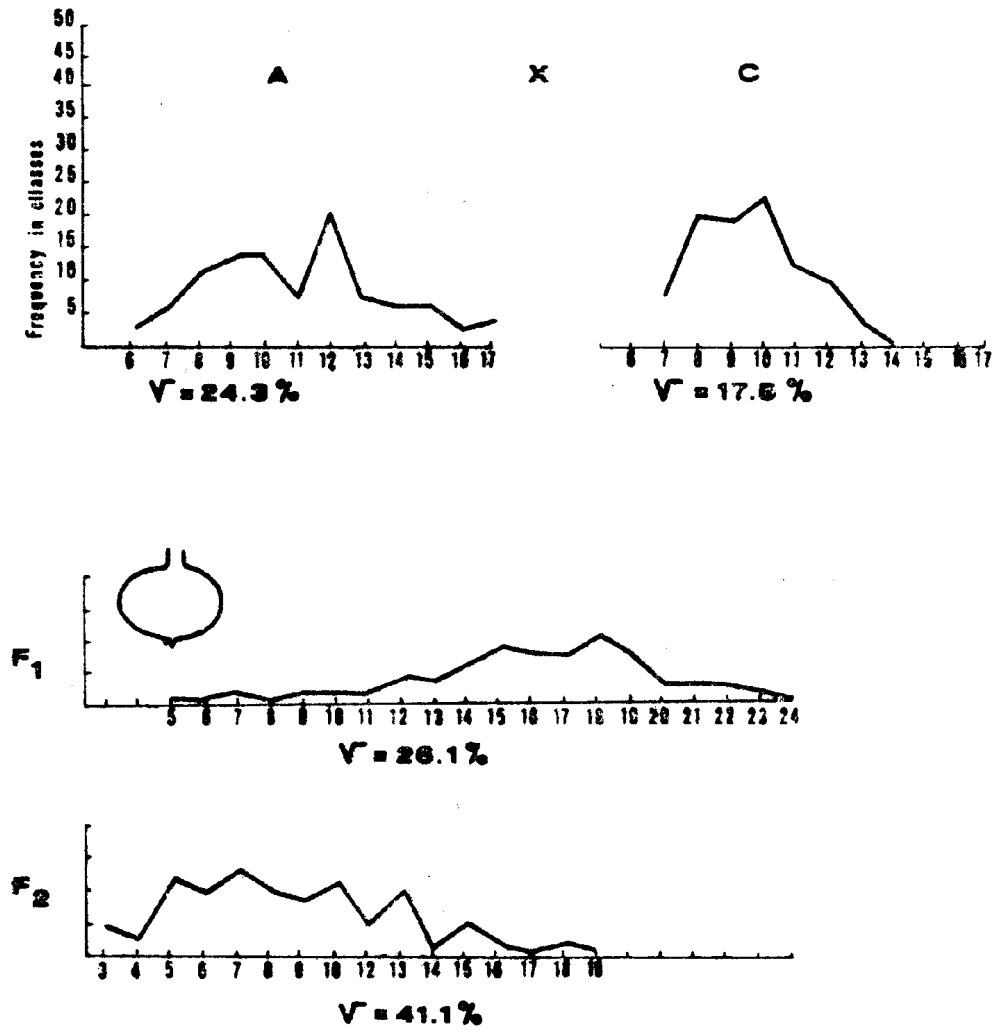

Fig. 2. Histogram of onion bulb weight variability of parental forms, $F_{1}$ and $F_{2}$ (A-16 $\times$ C-177). 1977. Classes in dag

The variability of traits in parental lines and in $\mathrm{F}_{1}$ and $\mathrm{F}_{2}$ is described by the standard deviation $\sigma$ and variability coefficient $\mathrm{V} \%$ where $\mathrm{V} \%=\frac{\sigma}{\mathrm{x}} \cdot 100 \%$.

The total variability in $F_{1}, F_{2}$ and parental components is presented as the phenotypic variability $\left(\sigma_{P}\right)$. The phenotypic variability is the sum of the variability caused by environmental factors $\left(\sigma_{\mathrm{E}}\right)$ and the effect of genes $\left(\sigma_{\mathrm{G}}\right)$ ( $\mathrm{F} \mathrm{a} \mathrm{l} \mathrm{c} \mathrm{o} \mathrm{n} \mathrm{e} \mathrm{r,} \mathrm{1960).} \mathrm{On} \mathrm{the} \mathrm{basis} \mathrm{of} \mathrm{measurements} \mathrm{of} \mathrm{traits}$ in $F_{2}$ hybrids and their parents, selected through partial inbreedings (under the same environmental conditions), the variability determined genetically was evaluated $\left(\sigma_{\mathrm{G}}=\sigma_{\mathrm{P}}-\sigma_{\mathrm{E}}\right)$ and heritability in the wide sense, without taking into accounts its elements bringing about changes in it, was determined. When the phenotypic variability $\left(\sigma_{\mathrm{P}}\right)$ and genetic 
Ta b le 10

Frequency distribution of the bulb shape of parental lines and hybrids

\begin{tabular}{|c|c|c|c|c|c|c|c|c|c|c|c|}
\hline \multirow[t]{2}{*}{ No. } & \multirow[t]{2}{*}{ Line and hybrid } & \multirow[t]{2}{*}{ Year } & \multicolumn{8}{|c|}{$\begin{array}{l}\text { Number of bulbs according } \\
\text { to the UPOV guideliness }\end{array}$} & \multirow[t]{2}{*}{ Quantity } \\
\hline & & & 12 & 3 & 4 & 5 & 6 & 7 & 8 & 9 & \\
\hline \multirow[t]{4}{*}{1.} & A- 6 & 1977 & -- & 72 & 24 & 59 & - & 5 & 5 & - & 100 \\
\hline & $C-177 a$ & 1977 & -6 & 50 & 41 & 3 & $\longrightarrow$ & - & $\ldots$ & - & 100 \\
\hline & $F_{1}(A-6 \times C-177 a)$ & 1977 & -- & 5 & 52 & 15 & 2 & 12 & 2 & - & 88 \\
\hline & $F_{2}(A-6 \times C-177 a)$ & 1977 & $2-$ & 50 & 16 & 8 & 1 & 23 & - & 一 & 100 \\
\hline \multirow[t]{7}{*}{2.} & A-16 & 1977 & -- & 7 & 18 & 71 & 一 & 2 & 2 & $\longrightarrow$ & 100 \\
\hline & & 1978 & --1 & 12 & 3 & 39 & 一 & 3 & 8 & - & 65 \\
\hline & $C-177 a$ & 1977 & -6 & 50 & 41 & 3 & - & - & 一 & 一 & 100 \\
\hline & & 1978 & -23 & 8 & - & - & - & 一 & - & - & 31 \\
\hline & $F_{1}(A-16 \times C-177 a)$ & 1977 & -1 & 3 & 29 & 16 & 7 & 22 & 3 & - & 80 \\
\hline & & 1978 & --1 & 12 & 5 & 16 & 一 & 59 & 4 & - & 96 \\
\hline & $F_{2}(A-16 \times C-177 a)$ & 1977 & $--?$ & 30 & 17 & 14 & 6 & 30 & 3 & - & 100 \\
\hline \multirow[t]{7}{*}{3.} & A-54 & 1977 & $-\cdots$ & - & 4 & 65 & - & 一 & 31 & 一 & 100 \\
\hline & & 1978 & - & 5 & 2 & 43 & - & 3 & 46 & 1 & 100 \\
\hline & $C-177 a$ & 1977 & -6 & 50 & 41 & 3 & - & 一 & 一 & - & 100 \\
\hline & & 1978 & -23 & 8 & - & - & - & 一 & - & - & 31 \\
\hline & $F_{1}(A-54 \times C-177 a)$ & 1977 & -- & 72 & 23 & 45 & - & 12 & 4 & - & 91 \\
\hline & & 1978 & -11 & 13 & 11 & 32 & 3 & 26 & 14 & - & 100 \\
\hline & $F_{2}(A-54 \times C-177 a)$ & 1977 & 513 & 371 & 16 & 3 & 17 & 18 & 3 & 一 & 100 \\
\hline \multirow[t]{3}{*}{4.} & A -58 & 1977 & -1 & 14 & 2 & 51 & - & 11 & 1 & 一 & 79 \\
\hline & $C-177 a$ & 1977 & -65 & 50 & 41 & 3 & - & 一 & - & - & 100 \\
\hline & $F_{1}(A-58 \times C-1770)$ & 1977 & -- & 8 & 5 & 一 & 11 & 43 & 4 & 1 & 72 \\
\hline \multirow[t]{7}{*}{5.} & $A-125$ & 1977 & -2 & 251 & 16 & 31 & - & 9 & 4 & - & 85 \\
\hline & & 1978 & --1 & 192 & 26 & 23 & 4 & 12 & 12 & 1 & 97 \\
\hline & C- $177 \mathrm{a}$ & 1977 & -65 & 50 & 41 & 3 & - & - & - & - & 100 \\
\hline & & 1978 & -23 & 8 & - & 一 & 一 & - & - & 一 & 31 \\
\hline & $F_{1}(A-125 \times C-177 a)$ & 1977 & -21 & 142 & 25 & 45 & 1 & 8 & 4 & 1 & 100 \\
\hline & & 1978 & $-\rightarrow$ & 6 & 7 & 4 & 1 & 1 & - & & 19 \\
\hline & $F_{2}(A-125 \times C-177 a)$ & 1977 & 324 & 441 & 19 & 10 & 8 & 12 & 2 & 一 & 100 \\
\hline \multirow[t]{3}{*}{6.} & A-19 & 1977 & -- & - & 3 & 1 & - & 17 & 46 & 一 & 67 \\
\hline & $C-177 a$ & 1977 & -65 & 504 & 41 & 3 & - & - & 一 & - & 100 \\
\hline & $F_{1}(A-19 \times C-177 a)$ & 1977 & -2 & 31 & 14 & 23 & 3 & 15 & 1 & - & 61 \\
\hline \multirow[t]{6}{*}{7.} & A-181 & 1977 & -- & - & 1 & 3 & 10 & 4 & 79 & 3 & 100 \\
\hline & & 1978 & -- & 2 & 9 & 6 & 24 & 25 & 32 & 2 & 100 \\
\hline & $C-177 a$ & 1977 & -65 & 504 & 41 & 3 & 一 & - & - & 一 & 100 \\
\hline & & 1978 & -23 & $8-$ & - & - & - & 一 & 一 & - & 31 \\
\hline & $F_{1}(A-181 \times C-177 a)$ & 1977 & -- & 42 & 21 & 27 & 7 & 22 & 18 & 1 & 100 \\
\hline & & 1978 & - 一 & 2 & 2 & 15 & 3 & 76 & 2 & - & 100 \\
\hline \multirow[t]{4}{*}{8.} & A-63 & 1977 & -- & 2 & 2 & 92 & - & 一 & 4 & - & 100 \\
\hline & $C-177 b$ & 1977 & --4 & 473 & 35 & 6 & - & 2 & - & 一 & 90 \\
\hline & $F_{1}(A-63 \times C-177 b)$ & 1977 & $1-$ & 31 & 12 & 43 & - & 36 & 5 & 一 & 100 \\
\hline & $F_{2}(A-63 \times C-177 b)$ & 1977 & 254 & 421 & 10 & 29 & 一 & 9 & 3 & - & 100 \\
\hline
\end{tabular}


Table 10 cont.

No. Line and hybrid $\quad \begin{gathered}\text { Number of bulbs according } \\ \text { to the UPOV guideliness }\end{gathered}$ Quantity

9. A-16

C-141

$F_{1}(\mathrm{~A}-16 \times \mathrm{C}-141)$

$\mathrm{F}_{2}(\mathrm{~A}-16 \times \mathrm{C}-141)$

10. A-58

C-141

$F_{1}(A-58 \times C-141)$

11. A-12

C-141

$\mathrm{F}_{1}(\mathrm{~A}-12 \times \mathrm{C}-141)$

12. A-125

C-181

$F_{1}(A-125 \times C-181)$

$\mathrm{F}_{2}(\mathrm{~A}-125 \times \mathrm{C}-181)$

13. A-181

C-181

$\mathrm{F}_{1}(\mathrm{~A}-181 \times \mathrm{C}-181)$

14. A-16

C-16

$F_{1}(\mathrm{~A}-16 \times \mathrm{C}-16)$

$\mathrm{F}_{2}(\mathrm{~A}-16 \times \mathrm{C}-16)$

15. A-6

C-6

$F_{1}(\mathrm{~A}-6 \times \mathrm{C}-6)$

$\mathrm{F}_{2}(\mathrm{~A}-6 \times \mathrm{C}-6)$

16. A-125

C-199

$\mathrm{F}_{1}(\mathrm{~A}-125 \times \mathrm{C}-199)$

$\mathrm{F}_{2}(\mathrm{~A}-125 \times \mathrm{C}-199)$

17. A-19

C-19

$\mathrm{F}_{1}(\mathrm{~A}-19 \times \mathrm{C}-19)$

$\mathrm{F}_{2}(\mathrm{~A}-19 \times \mathrm{C}-19)$
$1977-71871-22-100$

$1977-100-\ldots \ldots 100$

$1977-\begin{array}{llllll}28 & 22 & 12 & 1 & 25 & 2\end{array}-90$

$\begin{array}{llllllllll}1977 & 4 & 13 & 28 & 5 & 4 & 8 & 22 & 6 & -90\end{array}$

$1977-14251-111-79$

$1978-20468-26-100$

$1977-100-\ldots-\ldots-100$

$1978 \quad 14 \quad 152 \ldots \ldots \ldots 7$

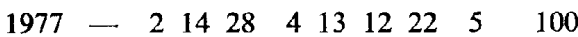

$19781115516-3--67$

$1978-129125 \ldots-\ldots-56$

$1978 \quad 14 \quad 152--\ldots--\ldots 67$

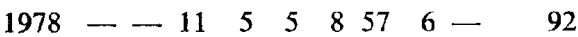

1977 - $251631-94-85$

$1978-\begin{array}{lllllllll}19 & 26 & 23 & 4 & 12 & 12 & 1 & 97\end{array}$

$\begin{array}{lllllllllll}1977 & 12 & 3 & 45 & 13 & 17 & 1 & 7 & 2 & - & 100\end{array}$

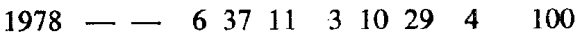

$1977 \quad 2-24 \quad 4520 \quad 4 \quad 4 \quad 1-100$

$19783-46111-327-100$

$1977 \quad 1 \quad 1303622 \quad 2 \quad 6 \quad 2-100$

1978 - $\begin{array}{lllllllll}2 & 2 & 9 & 6 & 24 & 25 & 32 & 2 & 100\end{array}$

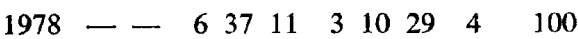

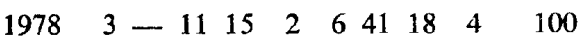

$1977--71871-22-100$

$1978-12339-38-65$

$1977-\begin{array}{llllllll}11 & 22 & 3 & 56 & 7 & 1 & \ldots & 100\end{array}$

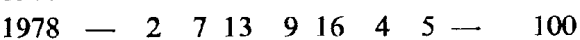

1977 - $\begin{array}{llllllll}6 & 20 & 18 & 7 & 18 & 27 & 4 & 100\end{array}$

$\begin{array}{llllllllll}1978 \quad 1-8 & 21 & 9 & 13 & 20 & 25 & 3 & 100\end{array}$

1977 - -23619655100

$1977-72459-55-100$

$1977-\ldots 171761-5-\ldots 100$

$\begin{array}{lllllllllll}1977 & 1 & 4 & 9 & 20 & 44 & 3 & 9 & 8 & 2 & 100\end{array}$

$1977-1281810151981100$

$1977-251631-94-85$

$1977-13253129-1 \quad 1-100$

1977 - $14322626-140$

$1977 \div 233401618 \div-100$

$1977-\ldots-31-1746-67$

$19776168-1-24--100$

$1977-\begin{array}{rllllllll}2 & 4 & 11 & 3 & 53 & 21 & 6 & 100\end{array}$

$\begin{array}{llllllllll}1977 \quad 1 & 18 & 2 & 34 & 1 & 26 & 18 & - & 100\end{array}$ 
Table 10 cont.

\begin{tabular}{|c|c|c|c|c|c|c|c|c|c|c|c|}
\hline \multirow[t]{2}{*}{ No. } & \multirow{2}{*}{ Line and hybrid } & \multirow{2}{*}{ Year } & \multicolumn{8}{|c|}{$\begin{array}{l}\text { Number of bulbs according } \\
\text { to the UPOV guideliness }\end{array}$} & \multirow[t]{2}{*}{ Quantity } \\
\hline & & & 1 & 3 & 4 & 5 & 6 & 7 & & 9 & \\
\hline \multirow[t]{3}{*}{18.} & A-16 & 1978 & -- & 12 & 3 & 39 & 一 & 3 & 8 & 一 & 65 \\
\hline & C-9 & 1978 & 1.26 & 40 & - & - & - & 一 & 一 & - & 67 \\
\hline & $F_{1}(A-16 \times C-9)$ & 1978 & $\cdots$ & 3 & 1 & 5 & 24 & 65 & 2 & - & 100 \\
\hline \multirow[t]{3}{*}{19.} & $A=101$ & 1978 & -- & 8 & 19 & 22 & 2 & 24 & 23 & 2 & 100 \\
\hline & $\mathrm{C}-9$ & 1978 & 126 & 40 & - & 一 & - & 一 & - & - & 67 \\
\hline & $F_{1}(A-101 \times C-9)$ & 1978 & $-\cdots$ & 8 & 一 & 3 & 8 & 5 & 2 & 一 & 26 \\
\hline \multirow[t]{3}{*}{20.} & $A-181$ & 1978 & -- & 2 & 9 & 6 & 24 & 25 & 32 & 2 & 10) \\
\hline & C-9 & 1978 & 126 & 40 & -- & 一 & - & 一 & $一$ & 一 & 67 \\
\hline & $F_{1}(\mathrm{~A}-181 \times \mathrm{C}-9)$ & 1978 & -- & - & 8 & 6 & 14 & 29 & 40 & 1 & 98 \\
\hline
\end{tabular}

T a ble 11

Frequency distribution of dry skin adherence of onion bulbs from parental lines and hybrids

\begin{tabular}{|c|c|c|c|c|c|c|c|c|c|}
\hline \multirow[t]{2}{*}{ No. } & \multirow[t]{2}{*}{ Line and hybrid } & \multirow[t]{2}{*}{ Year } & \multicolumn{3}{|c|}{$\begin{array}{l}\text { Number of bulbs } \\
\text { according to their } \\
\text { degrees of evaluation }\end{array}$} & \multirow[t]{2}{*}{$\begin{array}{c}\text { Quan- } \\
\text { tity }\end{array}$} & \multirow[t]{2}{*}{ Mean } & \multirow[t]{2}{*}{$\sigma$} & \multirow[t]{2}{*}{$V \%$} \\
\hline & & & 1 & 2 & 3 & & & & \\
\hline \multirow[t]{4}{*}{1.} & A- 6 & 1977 & $一$ & 21 & 79 & 100 & 2.79 & 0.46 & 16.5 \\
\hline & $C-177 a$ & 1977 & - & 3 & 97 & 100 & 2.97 & 0.18 & 6.1 \\
\hline & $F_{1}(A-6 \times C-177 a)$ & $\cdots \quad 1977$ & - & 9 & 79 & 88 & 2.90 & 0.32 & 11.0 \\
\hline & $F_{2}(A-6 \times C-177 a)$ & 1977 & 22 & 34 & 44 & 100 & 2.22 & 1.11 & 50.0 \\
\hline \multirow[t]{2}{*}{2.} & A-16 & 19.77 & 1 & 5 & 94 & 100 & 2.93 & 0.30 & 10.2 \\
\hline & & 1978 & 9 & 12 & 44 & 65 & 2.54 & 0.87 & 34.3 \\
\hline : & C-177a & 1977 & - & 3 & 97 & 100 & 2.97 & 018 & 6.1 \\
\hline & $\therefore$ & 1978 & 一 & 4 & 27 & 31 & 2.87 & 0.37 & 12.9 \\
\hline & $F_{1}(A-16 \times C-177 a)$ & 1977 & - & 6 & 74 & 80 & 2.92 & 0.25 & 8.6 \\
\hline & & 1978 & - & 19 & 77 & 96 & 2.80 & 0.45 & 16.1 \\
\hline & $F_{2}(A-16 \times C-177 a)$ & 1977 & 7 & 13 & 80 & 100 & 2.73 & 0.64 & 23.4 \\
\hline \multirow[t]{7}{*}{3.} & A-54 & 1977 & 1 & 35 & 64 & 100 & 2.63 & 0.63 & 23.9 \\
\hline & & 1978 & 16 & 43 & 41 & 100 & 2.25 & 0.76 & 33.8 \\
\hline & C-177a & 1977 & - & 3 & 97 & 100 & 2.97 & 0.18 & 6.1 \\
\hline & & 1978 & - & 4 & 27 & 31 & 2.87 & 0.37 & 12.9 \\
\hline & $F_{1}(A-54 \times C-177 a)$ & 1977 & 2 & 22 & 67 & 91 & 2.71 & 0.58 & 21.4 \\
\hline & & 1978 & - & 26 & 74 & 100 & 2.74 & 0.51 & 18.6 \\
\hline & $F_{2}(A-54 \times C-177 a)$ & 1977 & 32 & 38 & 30 & 100 & 1.98 & 0.79 & 39.9 \\
\hline \multirow[t]{3}{*}{4.} & A-58 & 1977 & 一 & 16 & 63 & 79 & 2.80 & 0.45 & 16.1 \\
\hline & C-177a & 1977 & 一 & 3 & 97 & 100 & 2.97 & 0.18 & 6.1 \\
\hline & $F_{1}(A-58 \times C-177 a)$ & 1977 & 1 & 24 & 47 & 72 & 2.64 & 0.63 & 23.9 \\
\hline \multirow[t]{2}{*}{5.} & A-125 & 1977 & - & 20 & 65 & 85 & 2.76 & 0.49 & 17.8 \\
\hline & & 1978 & 3 & 15 & 79 & 97 & 2.78 & 0.53 & 19.1 \\
\hline
\end{tabular}


Table 11 cont.

No. Line and hybrid $\quad$ Year $\begin{aligned} & \text { Number of bulbs } \\ & \begin{array}{l}\text { according to their Quan- Mean } \\ \text { degrees of evaluation tity }\end{array}\end{aligned}$

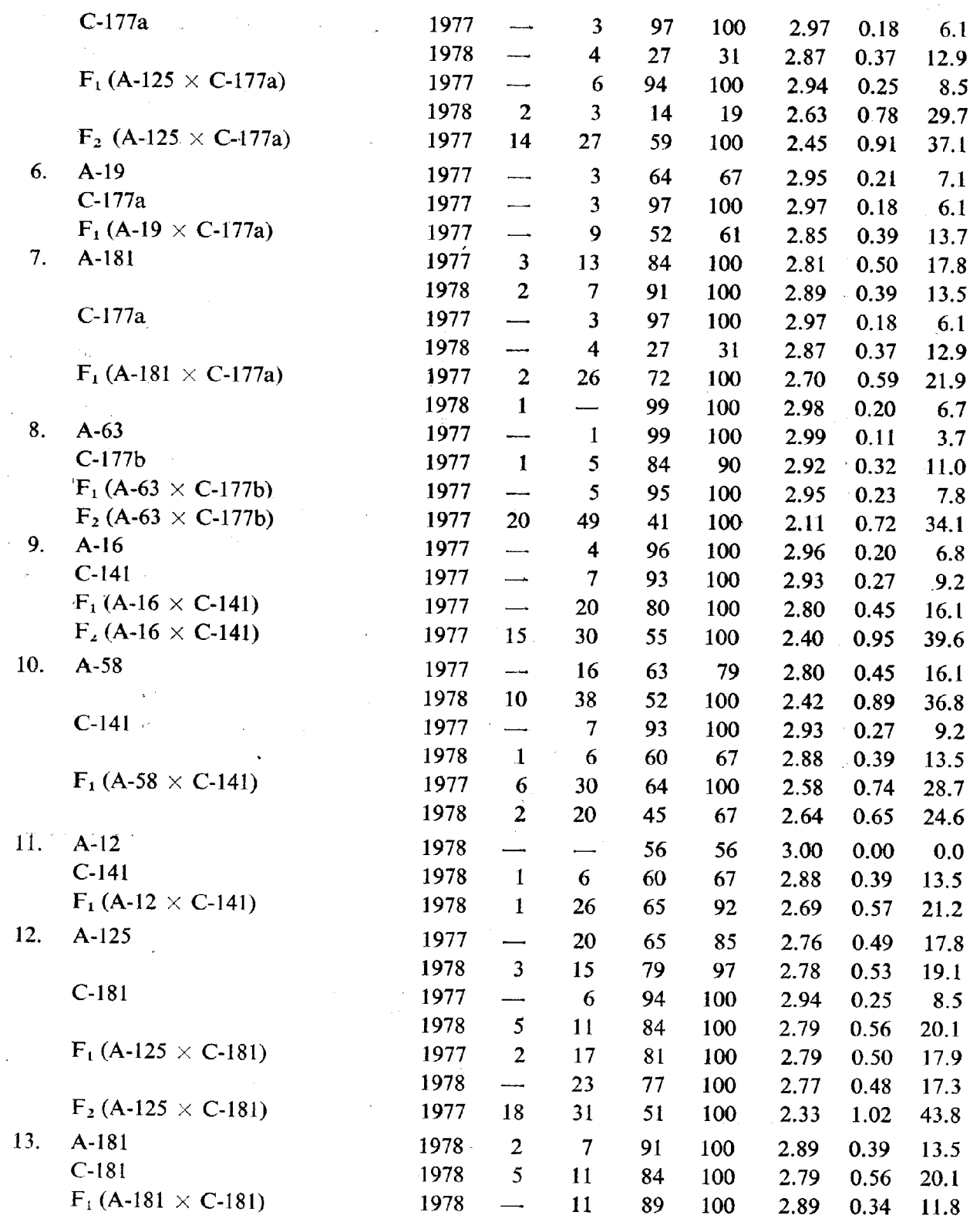


Table 11 cont.

\begin{tabular}{|c|c|c|c|c|c|c|c|c|c|}
\hline \multirow[t]{2}{*}{ No. } & \multirow[t]{2}{*}{ Line and hybrid } & \multirow[t]{2}{*}{ Year } & \multicolumn{3}{|c|}{$\begin{array}{l}\text { Number of bulbs } \\
\text { according to their } \\
\text { degrees of evaluation }\end{array}$} & \multirow[t]{2}{*}{$\begin{array}{l}\text { Quan- } \\
\text { tity }\end{array}$} & \multirow[t]{2}{*}{ Mean } & \multirow[t]{2}{*}{$\sigma$} & \multirow[t]{2}{*}{$\vee \%$} \\
\hline & & & 1 & 2 & 3 & & & & \\
\hline \multirow[t]{7}{*}{14.} & A-16 & 1977 & 1 & 5 & 94 & 100 & 2.93 & 0.30 & 10.2 \\
\hline & & 1978 & 9 & 12 & 44 & 65 & 2.54 & 0.87 & 34.3 \\
\hline & C-16 & 1977 & 一 & 16 & 84 & 100 & 2.84 & 0.40 & 14.1 \\
\hline & & 1978 & 1 & 6 & 93 & 100 & 2.92 & 0.32 & 11.0 \\
\hline & $F_{1}(A-16 \times C-16)$ & 1977 & 一 & 20 & 80 & 100 & 2.80 & 0.45 & 16.1 \\
\hline & & 1978 & 6 & 4 & 90 & 100 & 2.84 & 0.53 & 18.7 \\
\hline & $F_{2}(A-16 \times C-16)$ & 1977 & 29 & 52 & 19 & 100 & 1.90 & 0.70 & 36.8 \\
\hline \multirow[t]{4}{*}{15.} & A.6 & 1977 & - & 21 & 79 & 100 & 2.79 & 0.46 & 16.5 \\
\hline & C-6 & 1977 & - & 4 & 96 & 100 & 2.96 & 0.20 & 6.8 \\
\hline & $F_{1}(A-6 \times C-6)$ & 1977 & - & 1 & 99 & 100 & 2.99 & 0.11 & 3.7 \\
\hline & $F_{2}(A-6 \times C-6)$ & 1977 & 9 & 35 & 56 & 100 & 2.47 & 0.85 & 34.4 \\
\hline \multirow[t]{4}{*}{16.} & A-125 & 1977 & - & 20 & 65 & 85 & 2.76 & 0.49 & 17.8 \\
\hline & C-199 & 1977 & - & 一 & 100 & 100 & 3.00 & 0.00 & 0,0 \\
\hline & $F_{1}(A-125 \times C-199)$ & 1977 & 1 & 5 & 74 & 80 & 2.91 & 0.34 & 11.7 \\
\hline & $F_{2}(A-125 \times C-199)$ & 1977 & 24 & 43 & 33 & 100 & 2.09 & 0.76 & 36.4 \\
\hline \multirow[t]{4}{*}{17.} & A-19 & 1977 & 一 & 3 & 64 & 67 & 2.95 & 0.21 & 7.1 \\
\hline & C-19 & 1977 & - & 4 & 96 & 100 & 2.96 & 0.20 & 6.8 \\
\hline & $F_{1}(A-19 \times C-19)$ & 1977 & 一 & 4 & 96 & 100 & 2.96 & 0.20 & 6.8 \\
\hline & $F_{2}(A-19 \times C-19)$ & 1977 & 2 & 22 & 76 & 100 & 2.74 & 0.55 & 20.1 \\
\hline \multirow[t]{3}{*}{18.} & A-16 & 1978 & 9 & 12 & 44 & 65 & 2.54 & 0.87 & 34.3 \\
\hline & C-9 & 1978 & - & 14 & 53 & 67 & 2.79 & 0.46 & 16.5 \\
\hline & $F_{1}(A-16 \times C-9)$ & 1978 & 3 & 16 & 81 & 100 & 2.78 & 0.53 & 19.1 \\
\hline \multirow[t]{3}{*}{19.} & A-101 & 1978 & 2 & 4 & 94 & 100 & 2.92 & 0.35 & 12.0 \\
\hline & C-9 & 1978 & - & 14 & 53 & 67 & 2.79 & 0.46 & 16.5 \\
\hline & $F_{1}(A-101 \times C-9)$ & $1978^{\circ}$ & 2 & 4 & 20 & 26 & 2.96 & 0.69 & 25.7 \\
\hline \multirow[t]{3}{*}{20.} & A-181 & 1978 & 2 & 7 & 91 & 100 & 289 & 0.39 & 13.5 \\
\hline & C-9 & 1978 & - & 14 & 53 & 67 & 2.79 & 0.46 & 16.5 \\
\hline & $F_{1}(A-181 \times C-9)$ & 1978 & 14 & 57 & 27 & 98 & 2.13 & 0.65 & 30.5 \\
\hline
\end{tabular}

Table 12

Frequency distribution of dry skin color of onion bulbs from parental lines and hybrids

\begin{tabular}{|c|c|c|c|c|c|c|c|c|c|c|c|c|}
\hline \multirow[t]{2}{*}{ No. } & \multirow[t]{2}{*}{ Line and hybrid } & \multirow[t]{2}{*}{ Year } & \multicolumn{6}{|c|}{$\begin{array}{c}\text { Color of dry skin according } \\
\text { to degrees } \\
\text { of evaluation } \\
\end{array}$} & \multirow[t]{2}{*}{ Quan- } & \multirow[t]{2}{*}{ Mean } & \multirow[t]{2}{*}{$\sigma$} & \multirow[t]{2}{*}{$V \%$} \\
\hline & & & 1 & 2 & 3 & 4 & 5 & 6 & & & & \\
\hline \multirow[t]{4}{*}{1.} & A-6 & 1977 & - & - & 13 & 56 & 30 & 1 & 100 & 4.19 & 0.69 & 16.5 \\
\hline & C-177a & 1977 & - & - & - & 3 & 81 & 16 & 100 & 5.13 & 0.44 & 8.6 \\
\hline & $F_{1}(A-6 \times C-177 a)$ & 1977 & - & - & - & 13 & 66 & 9 & 88 & 4.95 & 0.50 & 10.1 \\
\hline & $F_{2}(A-6 \times C-177 a)$ & 1977 & 1 & - & 15 & 55 & 24 & 5 & 100 & 4.16 & 0.83 & 20.0 \\
\hline
\end{tabular}


Table 12 cont.

\begin{tabular}{|c|c|c|c|c|c|c|c|c|c|c|c|c|}
\hline \multirow[t]{2}{*}{ No. } & \multirow[t]{2}{*}{ Line and hybrid } & \multirow[t]{2}{*}{ Year } & \multicolumn{6}{|c|}{$\begin{array}{c}\text { Color of dry skin according } \\
\text { to degrees } \\
\text { of evaluation }\end{array}$} & \multirow[t]{2}{*}{$\begin{array}{c}\text { Quan- } \\
\text { tity }\end{array}$} & \multirow[t]{2}{*}{ Mean } & \multirow[t]{2}{*}{$\sigma$} & \multirow[t]{2}{*}{$V \%$} \\
\hline & & & 1 & 2 & 3 & 4 & 5 & 6 & & & & \\
\hline \multirow[t]{7}{*}{2.} & A-16 & 1977 & - & 一 & 8 & 42 & 45 & 5 & 100 & 4.47 & 0.89 & 19.9 \\
\hline & & 1978 & 一 & - & 29 & 23 & 13 & - & 65 & 3.75 & 1.08 & 28.8 \\
\hline & C-177a & 1977 & $\rightarrow$ & - & - & 3 & 81 & 16 & 100 & 5.13 & 0.44 & 8.6 \\
\hline & & 1978 & - & 一 & 4 & 10 & 17 & - & 31 & 4.42 & 0.93 & 21.0 \\
\hline & $F_{1}(A-16 \times C-177 a)$ & 1977 & - & 一 & - & 9 & 66 & 5 & 80 & 4.95 & 0.42 & 8.5 \\
\hline & & 1978 & 一 & - & 42 & 53 & 1 & - & 96 & 3.57 & 0.67 & 18.8 \\
\hline & $F_{2}(A-16 \times C-177 a)$ & 1977 & - & - & 8 & 35 & 45 & 12 & 100 & 4.61 & 0.89 & 19.3 \\
\hline \multirow[t]{6}{*}{3.} & $A-54$ & 1977 & - & - & 32 & 57 & 10 & 1 & 100 & 3.80 & 0.68 & 17.9 \\
\hline & C $-177 \mathrm{a}$ & $\begin{array}{l}1978 \\
1977\end{array}$ & - & - & 51 & $\begin{array}{r}48 \\
3\end{array}$ & $\begin{array}{r}1 \\
81\end{array}$ & $\overline{16}$ & $\begin{array}{l}100 \\
100\end{array}$ & $\begin{array}{l}3.50 \\
5.13\end{array}$ & $\begin{array}{l}0.72 \\
0.44\end{array}$ & $\begin{array}{r}20.6 \\
8.6\end{array}$ \\
\hline & & 1978 & - & - & 4 & 10 & 17 & - & 31 & 4.42 & 0.93 & 21.0 \\
\hline & $F_{1}(A-54 \times C-177 a)$ & 1977 & - & - & 3 & 26 & 58 & 4 & 91 & 4.69 & 0.68 & 14.5 \\
\hline & & 1978 & - & - & 26 & 70 & 4 & - & 100 & 3.78 & 0.55 & 14.6 \\
\hline & $F_{2}(A-54 \times C-177 a)$ & 1977 & 2 & 一 & 43 & 42 & 13 & - & 100 & 3.64 & 1.01 & 27.8 \\
\hline \multirow[t]{3}{*}{4.} & A-58 & 1977 & $\ldots$ & - & 21 & 45 & 12 & 1 & 79 & 3.91 & 0.68 & 17.4 \\
\hline & C- $177 \mathrm{a}$ & 1977 & - & - & - & 3 & 81 & 16 & 100 & 5.13 & 0.44 & 8.6 \\
\hline & $F_{1}(A-58 \times C-177 a)$ & 1977 & 一 & - & 17 & 49 & 5 & 1 & 72 & 3.86 & 0.60 & 15.5 \\
\hline \multirow[t]{7}{*}{5.} & A-125 & 1977 & - & - & 2 & 27 & 56 & $\longrightarrow$ & 85 & 4.63 & 0.64 & 13.8 \\
\hline & & 1978 & - & - & 29 & 48 & 20 & - & 97 & 3.91 & 0.72 & 18.4 \\
\hline & $C-177 a$ & 1977 & - & - & - & 3 & 81 & 16 & 100 & 5.13 & 0.44 & 8.6 \\
\hline & & 1978 & - & $\ldots$ & 4 & 10 & 17 & - & 31 & 4.42 & 0.93 & 21.0 \\
\hline & $F_{1}(A-125 \times C-177 a)$ & 1977 & 一 & - & 7 & 41 & 44 & 8 & 100 & 4.53 & 0.88 & 19.4 \\
\hline & & 1978 & 6 & - & 6 & 3 & 4 & - & 19 & 2.95 & 1.54 & 52.2 \\
\hline & $F_{2}(A-125 \times C-177 a)$ & 1977 & 6 & 一 & 29 & 38 & 23 & 4 & 100 & 3.84 & 0.83 & 21.6 \\
\hline \multirow[t]{3}{*}{6.} & A-19 & 1977 & - & $一$ & - & 2 & 56 & 9 & 67 & 5.10 & 0.41 & 8.0 \\
\hline & $C-177 a$ & 1977 & - & - & - & 3 & 81 & 16 & 100 & 5.13 & 0.44 & 8.6 \\
\hline & $F_{2}(A-19 \times C-177 a)$ & 1977 & - & 一 & 一 & 10 & 47 & 4 & 61 & 4.90 & 0.48 & 9.8 \\
\hline \multirow[t]{6}{*}{7.} & $A-181$ & 1977 & - & - & 4 & 10 & 57 & 29 & 100 & 5.11 & 0.74 & 14.5 \\
\hline & & 1978 & 一 & - & - & 21 & 76 & 3 & 100 & 4.82 & 0.49 & 10.2 \\
\hline & C-177a & 1977 & 一 & - & 一 & 3 & 81 & 16 & 100 & 5.13 & 0.44 & 8.6 \\
\hline & & 1978 & - & 一 & 4 & 10 & 17 & - & 31 & 4.42 & 0.93 & 21.0 \\
\hline & $F_{1}(A-18 \times C-177 a)$ & 1977 & - & - & 3 & 46 & 45 & 6 & 100 & 4.54 & 0.85 & 18.7 \\
\hline & & 1978 & - & - & 一 & 6 & 94 & - & 100 & 4.94 & 0.25 & 5.1 \\
\hline \multirow[t]{4}{*}{8.} & A-63 & 1977 & - & - & - & 8 & 91 & 1 & 100 & 4.93 & 0.30 & 6.1 \\
\hline & $C-177 b$ & 1977 & 一 & - & 3 & 37 & 39 & 11 & 90 & 4.64 & 0.82 & 17.7 \\
\hline & $F_{1}(A-63 \times C-177 b)$ & 1977 & 1 & - & 10 & 60 & 28 & 1 & 100 & 4.17 & 0.72 & 17.3 \\
\hline & $F_{2}(A-63 \times C-177 b)$ & 1977 & 一 & - & 9 & 56 & 27 & 8 & 100 & 4.34 & 0.83 & 19.1 \\
\hline \multirow[t]{4}{*}{9.} & A-16 & 1977 & - & - & 8 & 42 & 45 & 5 & 100 & 4.47 & 0.89 & 19.9 \\
\hline & $C-141$ & 1977 & - & 一 & 58 & 42 & - & - & 100 & 3.42 & 0.65 & 19.0 \\
\hline & $F_{1}(A-16 \times C-141)$ & 1977 & - & $\ldots$ & 28 & 47 & 14 & 1 & 90 & 3.87 & 0.72 & 18.6 \\
\hline & $F_{2}(A-16 \times C-141)$ & 1977 & - & - & 14 & 40 & 31 & 5 & 90 & 4.30 & 0.85 & 19.8 \\
\hline \multirow[t]{2}{*}{10.} & A-58 & 1977 & - & 一 & 21 & 45 & 12 & 1 & 79 & 3.91 & 0.68 & 17.4 \\
\hline & & 1978 & - & 一 & 48 & 32 & 20 & - & 100 & 3.72 & 1.06 & 28.5 \\
\hline
\end{tabular}


Table 12 cont.

\begin{tabular}{|c|c|c|c|c|c|c|c|c|c|c|c|c|}
\hline \multirow[t]{2}{*}{ No. } & \multirow[t]{2}{*}{ Line and hybrid } & \multirow[t]{2}{*}{ Year } & \multicolumn{6}{|c|}{$\begin{array}{c}\text { Color of dry skin according } \\
\text { to degrees } \\
\text { of evaluation }\end{array}$} & \multirow[t]{2}{*}{$\begin{array}{c}\text { Quan- } \\
\text { tity }\end{array}$} & \multirow[t]{2}{*}{ Mean } & \multirow[t]{2}{*}{$\sigma$} & \multirow[t]{2}{*}{$V \%$} \\
\hline & & & 1 & 2 & 3 & 4 & 5 & 6 & & & & \\
\hline \multirow{7}{*}{11.} & C-141 & 1977 & - & - & 58 & 42 & - & - & 100 & 3.42 & 0.65 & 19.0 \\
\hline & & 1978 & - & 一 & 33 & 31 & 3 & - & 67 & 3.55 & 0.81 & 22.8 \\
\hline & $F_{1}(A-58 \times C-141)$ & 1977 & - & - & 12 & 44 & 42 & 2 & 100 & 4.34 & 0.79 & 18.2 \\
\hline & & 1978 & - & 一 & 22 & 38 & 7 & - & 67 & 3.78 & 0.65 & 17.2 \\
\hline & A-12 & 1978 & 一 & 一 & - & 13 & 43 & - & 56 & 4.77 & 0.48 & 10.1 \\
\hline & $C-141$ & 1978 & - & 一 & 33 & 31 & 3 & - & 67 & 3.55 & 0.81 & 22.8 \\
\hline & $F_{1}(A-12 \times C-141)$ & 1978 & 1 & - & 47 & 44 & - & 一 & 92 & 3.46 & 0.73 & 21.1 \\
\hline \multirow[t]{7}{*}{12.} & A-125 & 1977 & - & - & 2 & 27 & 56 & - & 85 & 4.63 & 0.64 & 13.8 \\
\hline & & 1978 & - & - & 29 & 48 & 20 & - & 97 & 3.91 & 0.72 & 18.4 \\
\hline & C-181 & 1977 & - & - & 41 & 46 & 10 & 3 & 100 & 3.75 & 0.80 & 21.3 \\
\hline & & 1978 & 一 & - & 3 & 21 & 71 & 5 & 100 & 4.78 & 0.2 & 13.0 \\
\hline & $F_{1}(A-125 \times C-181)$ & 1977 & - & - & 31 & 44 & 25 & - & 100 & 3.94 & 0.75 & 19.0 \\
\hline & & 1978 & - & 一 & 52 & 43 & 5 & - & 100 & 3.53 & 0.80 & 22.7 \\
\hline & $F_{2}(A-125 \times C-181)$ & 1977 & 12 & - & 26 & 37 & 20 & 5 & 100 & 3.68 & 1.32 & 35.9 \\
\hline \multirow[t]{3}{*}{13.} & A-181 & 1978 & 一 & - & 一 & 21 & 76 & 3 & 100 & 4.82 & 0.49 & 10.2 \\
\hline & $C-181$ & 1978 & - & - & 3 & 21 & 71 & 5 & 100 & 4.78 & 0.62 & 13.0 \\
\hline & $F_{1}(A-181 \times C-181)$ & 1978 & - & 一 & 41 & 53 & 6 & - & 100 & 3.65 & 0.69 & 18.9 \\
\hline \multirow[t]{7}{*}{14.} & A-16 & 1977 & - & - & 8 & 42 & 45 & 5 & 100 & 4.47 & 0.89 & 19.9 \\
\hline & & 1978 & - & 一 & 29 & 23 & 13 & - & 65 & 3.75 & 1.08 & 28.8 \\
\hline & C-16 & 1977 & - & - & - & 一 & 31 & 69 & 100 & 5.69 & 0.56 & 9.8 \\
\hline & & 1978 & - & 一 & 6 & 36 & 58 & - & 100 & 4.52 & 0.78 & 17.3 \\
\hline & $F_{1}(\mathrm{~A}-16 \times \mathrm{C}-16)$ & 1977 & $\cdots$ & - & 24 & 58 & 17 & 1 & 100 & 3.95 & 0.67 & 17.0 \\
\hline & & 1978 & 1 & - & 35 & 49 & 15 & - & 100 & 3.77 & 0.77 & 20.4 \\
\hline & $F_{2}(A-16 \times C-16)$ & 1977 & 1 & - & 35 & 54 & 10 & - & 100 & 3.72 & 0.74 & 19.9 \\
\hline \multirow[t]{4}{*}{15.} & A-6 & 1977 & - & - & 13 & 56 & 30 & 1 & 100 & 4.19 & 0.69 & 16.5 \\
\hline & C-6 & 1977 & - & - & - & 4 & 90 & 6 & 100 & 5.02 & 0.32 & 6.4 \\
\hline & $F_{1}(A-6 \times C-6)$ & 1977 & - & - & - & 40 & 59 & 1 & 100 & 4.61 & 0.64 & 13.9 \\
\hline & $F_{2}(A-6 \times C-6)$ & 1977 & - & - & 1 & 30 & 46 & 23 & 100 & 4.91 & 0.76 & 15.5 \\
\hline \multirow[t]{4}{*}{16.} & A-125 & 1977 & - & - & 2 & 27 & 56 & - & 85 & 4.63 & 0.64 & 13.8 \\
\hline & C-199 & 1977 & - & 一 & - & 9 & 85 & 6 & 100 & 4.97 & 0.39 & 7.9 \\
\hline & $F_{1}(A-125 \times C-199)$ & 1977 & - & - & - & 16 & 61 & 3 & 80 & 4.84 & 0.49 & 10.1 \\
\hline & $\mathrm{F}_{2}(\mathrm{~A}-125 \times \mathrm{C}-199)$ & 1977 & - & - & 8 & 44 & 46 & 2 & 100 & 4.42 & 0.89 & 20.1 \\
\hline \multirow[t]{4}{*}{17.} & $\mathrm{~A}-19$ & 1977 & - & 一 & - & 2 & 56 & 9 & 67 & 5.10 & 0.41 & 8.0 \\
\hline & C-19 & 1977 & - & - & 14 & 57 & 26 & 3 & 100 & 4.18 & 0.72 & 17.2 \\
\hline & $F_{1}(A-19 \times C-19)$ & 1977 & $\longrightarrow$ & 2 & 14 & 57 & 27 & - & 100 & 4.09 & 0.70 & 17.1 \\
\hline & $\begin{array}{l}F_{2}(A-19 \times C-19) \\
A-16\end{array}$ & $\begin{array}{l}1977 \\
1978\end{array}$ & 1 & - & $\begin{array}{l}35 \\
29\end{array}$ & $\begin{array}{l}43 \\
23\end{array}$ & $\begin{array}{l}19 \\
13\end{array}$ & $\underline{2}$ & $\begin{array}{r}100 \\
65\end{array}$ & $\begin{array}{l}3.85 \\
3.75\end{array}$ & $\begin{array}{l}0.85 \\
1.08\end{array}$ & $\begin{array}{l}22.1 \\
28.8\end{array}$ \\
\hline \multirow{2}{*}{18.} & C-9 & 1978 & - & 一 & 一 & - & 67 & - & 67 & 5.00 & 0.00 & 0.0 \\
\hline & $F_{1}(A-16 \times C-9)$ & 1978 & - & - & 55 & 39 & 6 & - & 100 & 3.51 & 0.80 & 22.8 \\
\hline \multirow[t]{3}{*}{19.} & $A-101$ & 1978 & - & 一 & 21 & 52 & 24 & 3 & 100 & 4.09 & 0.76 & 18.6 \\
\hline & C-9 & 1978 & - & - & - & - & 67 & - & 67 & 5.00 & 0.00 & 0.0 \\
\hline & $\mathrm{F}_{1}(\mathrm{~A}-101 \times \mathrm{C}-9)$ & 1978 & - & - & 1 & 13 & 12 & 一 & 26 & 4.42 & 0.72 & 16.3 \\
\hline \multirow[t]{3}{*}{20.} & A-191 & 1978 & - & - & 一 & 21 & 76 & 3 & 100 & 4.82 & 0.49 & 10.2 \\
\hline & C-9 & 1978 & 一 & 一 & - & - & 67 & - & 67 & 5.00 & 0.00 & 0.0 \\
\hline & $F_{1}(A-181 \times C-9)$ & 1978 & 1 & - & 45 & 47 & 4 & 1 & 98 & 3.57 & 0.80 & 22.4 \\
\hline
\end{tabular}


variability $\left(\sigma_{G}\right)$ were ascertained, the heritability index was computed, using the formula (Mahmud and Kramer, 1951):

$$
\mathrm{h}^{2}=\frac{\mathrm{Q}_{\mathrm{F}_{2}}^{2}-\sqrt{\mathrm{Q}_{\mathrm{A}}^{2} \times \mathrm{Q}_{\mathrm{C}}^{2}}}{\mathrm{Q}_{\mathrm{F} 2}} \cdot 100
$$

\section{RESULTS}

The results of these studies on the variability of traits are compiled in Tables (3-11). However, because of space limitations, several details concerning, among others, variability, heve been omitted from these tables. In addition, the histograms from two representative parental lines, A-16 and C-177, and the $F_{1}$ and $F_{2}$ hybrids obtained from them (Figs. 2-8) are presented as examples.

The highest variability in most of the $F_{1}$ hybrids was shown by bulb weight (Table 3, Fig. 2) and by dry skin thickness (Table 7, Fig. 6) and, next in order, by collar thickness (Table 6, Fig. 5), bulb diameter (Table 5, Fig. 4) and root disc diameter (Table 9, Fig. 8). Bulb firmness exhibited the lowest variability (Table 8, Fig. 7) as did bulb height (Table 4, Fig. 3).

Low variability and good uniformity of bulb traits were found in several parental lines (A-63, C-6, C-141 and C-177), which indicates that they were homozygous to a high degree.

The variability of the studied traits in the parental lines was low, whereas in $F_{1}$ hybrids, it was higher, but concentrated in several central classes. Variability of traits in $F_{2}$ was clearly high, as indicated by flattened histograms and distinct inflection of the line due to segregation of traits and intermediate values or completely new ones, not seen in the parents, being found (Tables 4, 5, 6 and 9, Figs. 3, 4, 5 and 8).

The segregation of traits in $F_{2}$ deserves special attention, because a drop in the bulb characteristics having great economical importance, such as bulb weight (Table 3, Fig. 2) and dry skin thickness (Table 7, Fig. 6) were observed. This is shown by the high number of small bulbs (having low weight) with thin skin, which is clearly seen of Fig. 2 and 6. These results point to the high value of the characteristics of $F_{1}$ hybrids, especially to their good uniformity wich makes them well suited for mechanical grading and more easily prepared for market handling. The percentage of market crop increases in this way, and the percentage of onions not suitable for export falls. Because of this, the reproduction of $F_{1}$ hybrid seeds is not worthwhile, because the market value of the onions drastically drops due to the segregation of traits in $\mathrm{F}_{2}$. 

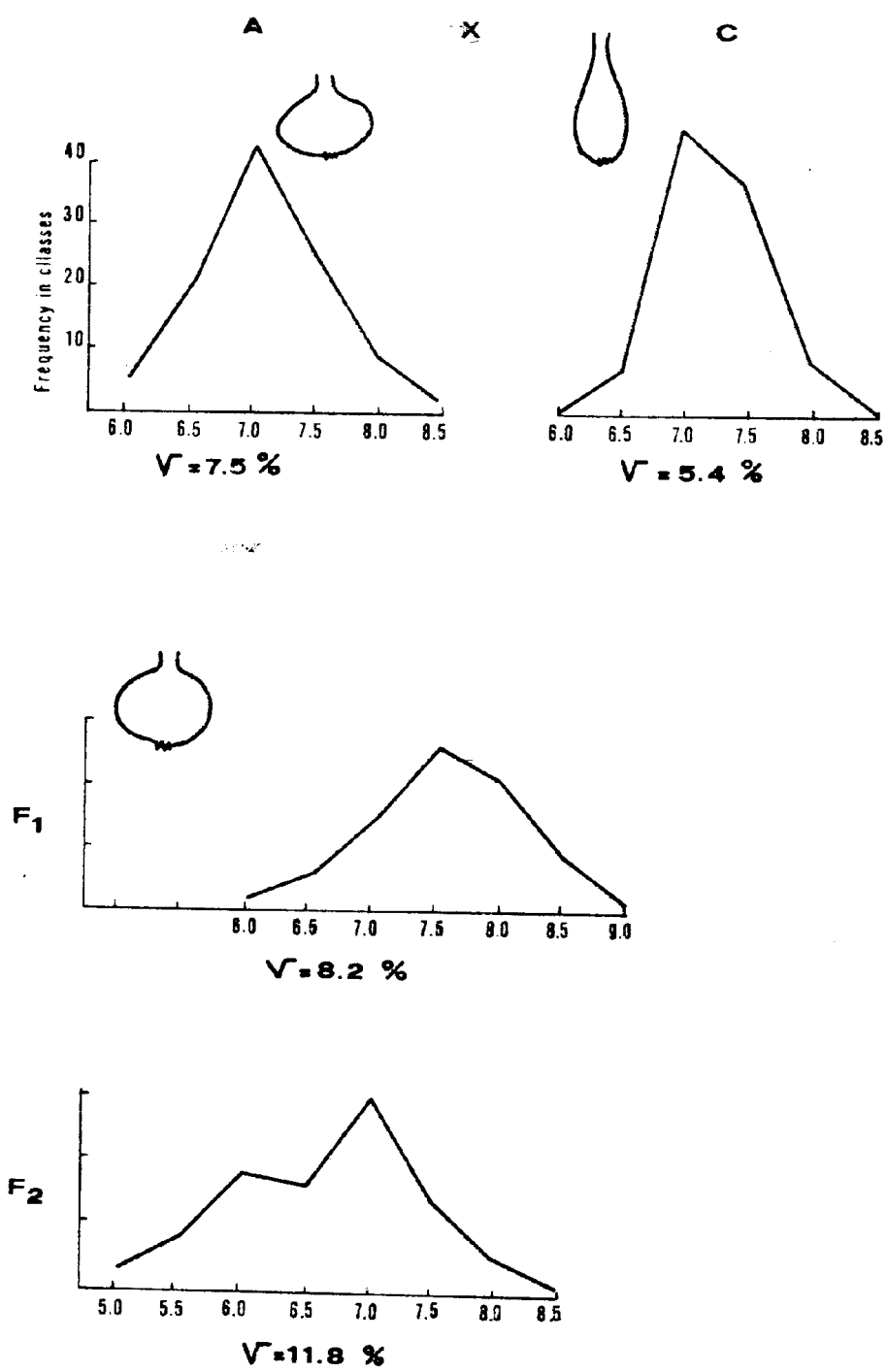

Fig. 3. Histogram of onion bulb height variability of parental forms, $\mathbf{F}_{1}$ and $\mathbf{F}_{\mathbf{2}}$ (A-16 $\times$ C-137). 1977. Classes in $\mathrm{cm}$

The appearance of several favorable traits in $F_{2}$, e.g. a thinner collar (Table 6, Fig. 5), not seen in the parental forms, is a very valuable phenomenon from the point of view of breeding. This creates the possibility of using this trait in breeding new cultivars onions with the valued thin neck and not having a tendency to develop a thick neck.

Analysis of the bulb shape, color and dry skin adherence, based on the analysis of the frequency distribution according to the UPOV 

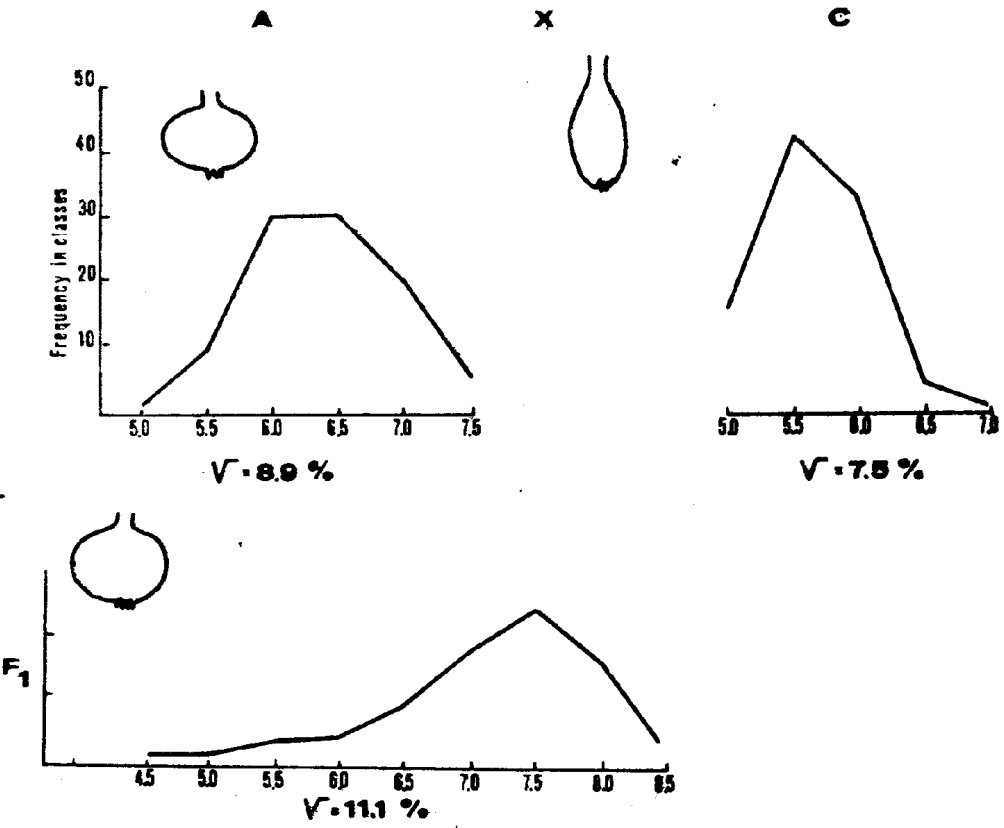

$r \cdot 7.8 \%$

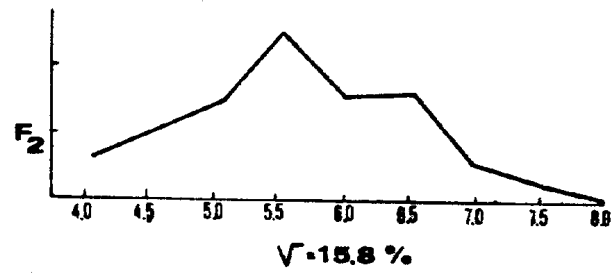

Fig. 4. Histogram of onion bulb diameter variability of parental forms, $F_{1}$ and $F_{2}$ $(\mathrm{A}-16 \times \mathrm{C}-177)$. Classes in $\mathrm{cm}$

classification, may be very useful in breeding due to the variability of the studied populations.

The bulb shape (Table 10) as determined by bonification, did not exhibit a normal distribution, and for this reason, it was not possible to conduct a statistical analysis. It was found that the elongated bulb shape (Fig. 1, Table 10) (elliptic or oval) in C lines was characterized by low variability and good equalization. Onion lines possessing this trait exhibited high stability in this respect.

The shape of bulbs in line A (Table 10, Fig. 1) in the studied material was close to shperical (broad ellipsoid, broad ovate, broad obovate, 


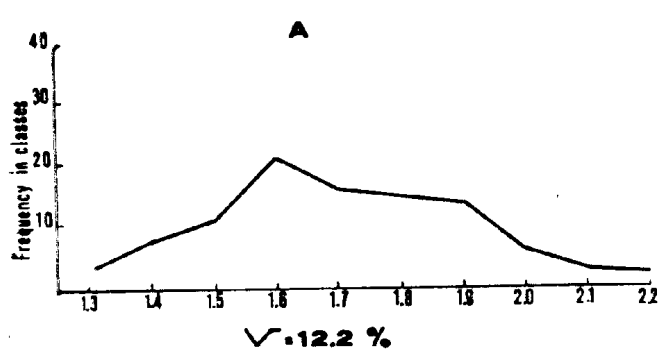

$\mathbf{x}$

c
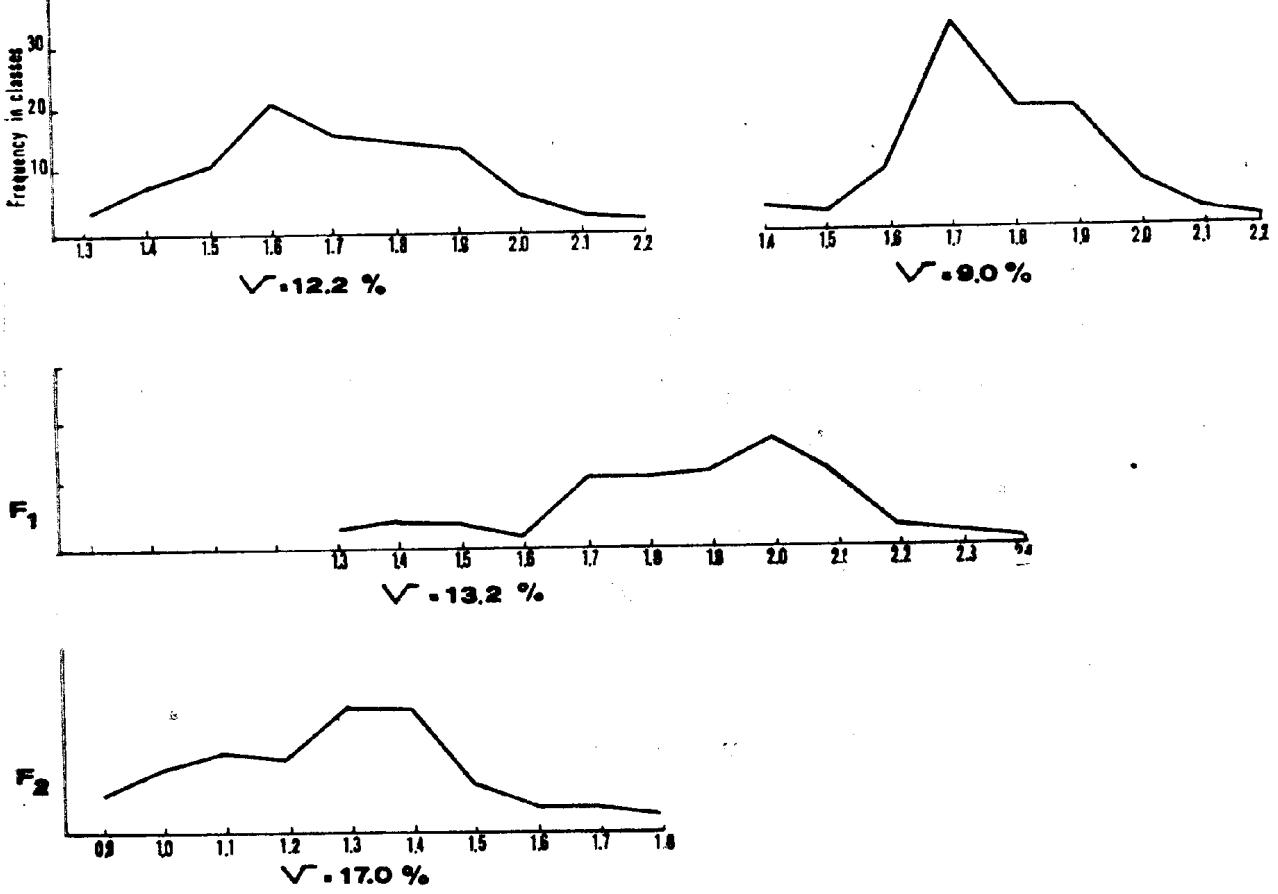

Fig. 5. Histogram of onion collar thickness variability of parental forms, $F_{1}$ and $F_{2}$ (A-16 $\times$ C-177). 1977. Classes in $\mathrm{cm}$

rhombic, transverse elliptic) (Table 10, Fig. 1) and characterized by a greater variability. The shape of the bulbs was easily influenced by changing atmospheric conditions, as seen under the influence of different weather in 1977 and 1978 (Table 1).

Thick collar (Table 6, Fig. 1) was typical for those lines having a bulb shape close to elliptic, although $F_{1}$ hybrids had a thinner collar than parents under different environmental conditions, which is a favorable trait from the point of view of breeding.

An outstanding root disc (Table 9, Fig. 1), was typical for parental lines with a more or less elongated shape.

The position of the root disc (Fig. 1) in line $A$ and in $F_{1}$ and $F_{2}$ hybrids was varied, and all three types (deep, flat and outstanding) were represented.

Most of the lines and $F_{1}$ hybrids had fairly well adhering dry skin, which is indicated by the high number of onions falling into class 3 (Table 11). The better adhesion of paternal line $\mathrm{C}$ dry skin had a clearly 

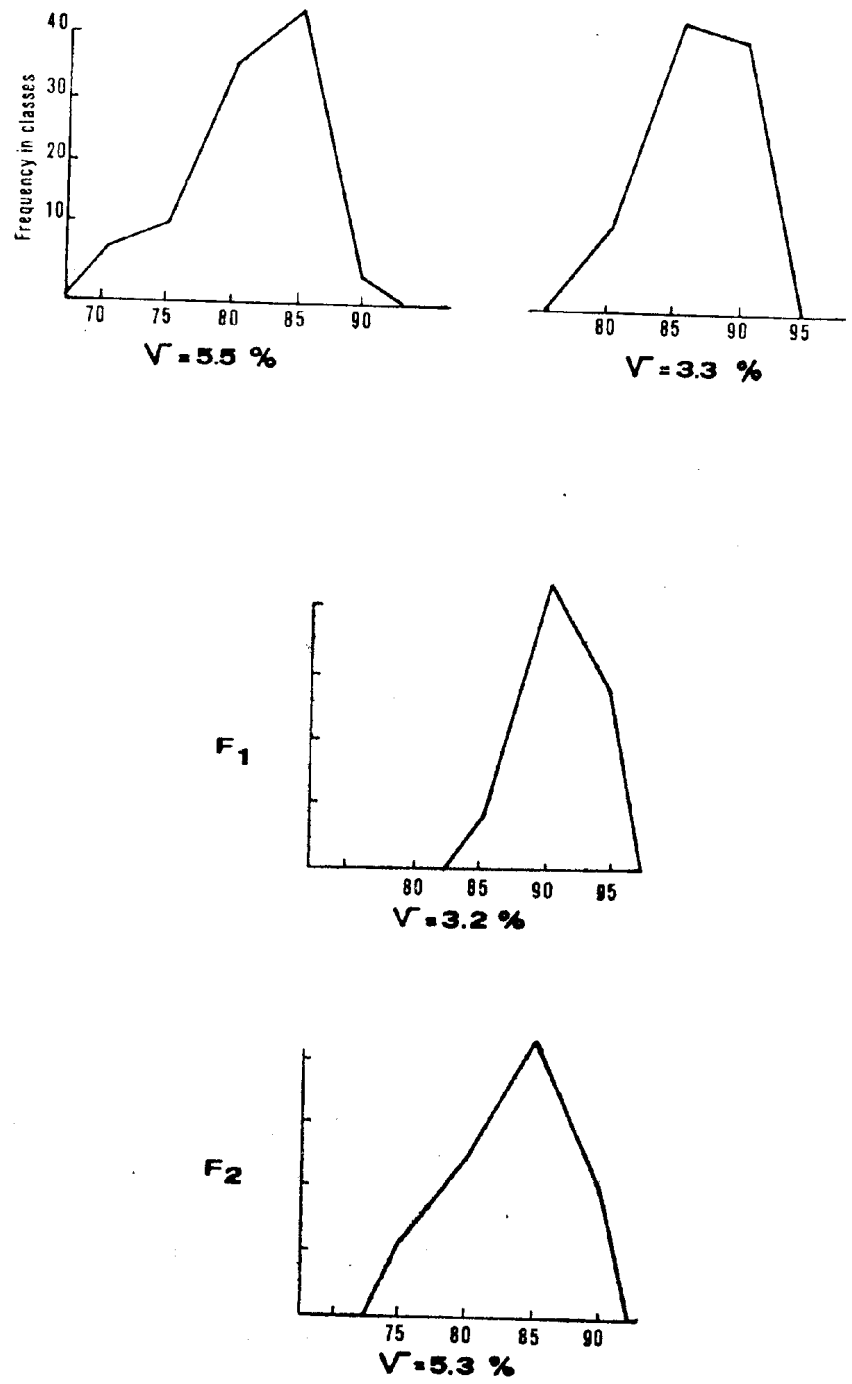

Fig. 6. Histogram of onion dry skin thickness variability of parental forms, $F_{1}$ and $\mathrm{F}_{2}$ (A-16 $\times$ C-177). 1977. Classes in $\mu$.

positive influence on the quality of dry skin in $F_{1}$ hybrids, in spite of the worse dry skin of the maternal forms. A partial dominance of the superior dry skin was therefore observed in $F_{1}$ hybrids. The lines and $F_{1}$ hybrids with the more weakly adhering dry skin were also characterized by a higher variability in respect to this trait. In addition to this, they were more sensitive to changing environmental conditions, which is 

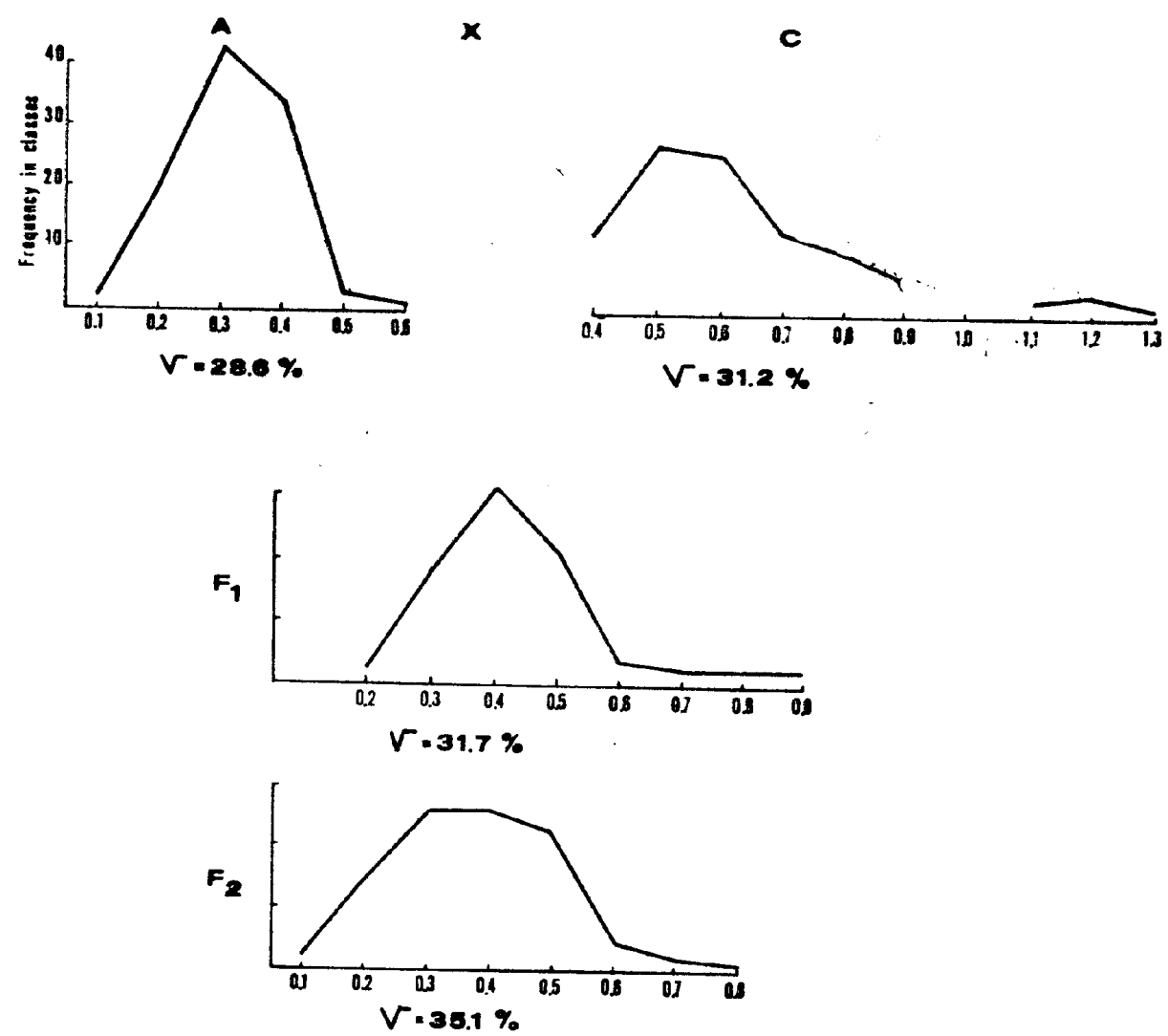

Fig. 7. Histogram of onion bulb firmness variability of parental forms, $F_{1}$ and $F_{2}$ (A-16 $\times$ C-177). 1977. In durometer readings

indicated by the different values of the variability coefficient in both years under study.

In order to obtain very strong and tightly adhering dry skin in $F_{1}$ hybrids, parental forms should be chosen which have well adhering dry skin. Then, its quality and stability, that is, lower sensitivity to environmental conditions, are better in $F_{1}$ hybrids.

In $F_{2}$ hybrids, the trait of dry skin adherence segregated in the direction of a significant decrease in its quality.

The color of the dry skin lines $\mathrm{A}$ and $\mathrm{C}$ ranged from straw colored to light straw colored (Table 12). The majority of the onions with dark dry skin in the mentioned lines and hybrids were classified in two neighboring classes, which points to their good uniformity in respect to this trait. The reaction of plants to changing environmental conditions was observed. The values of the variability coefficients for this trait for 


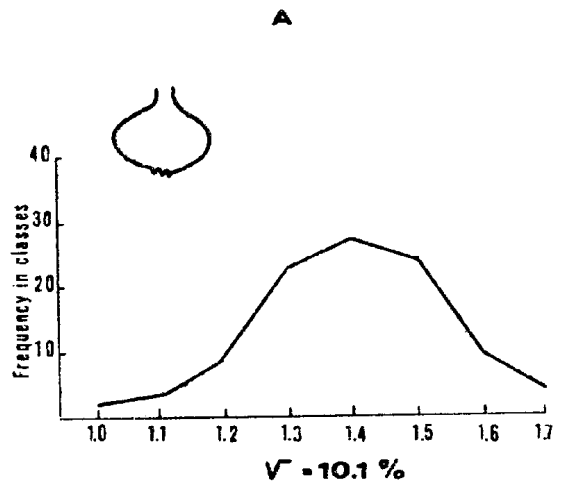

$x$
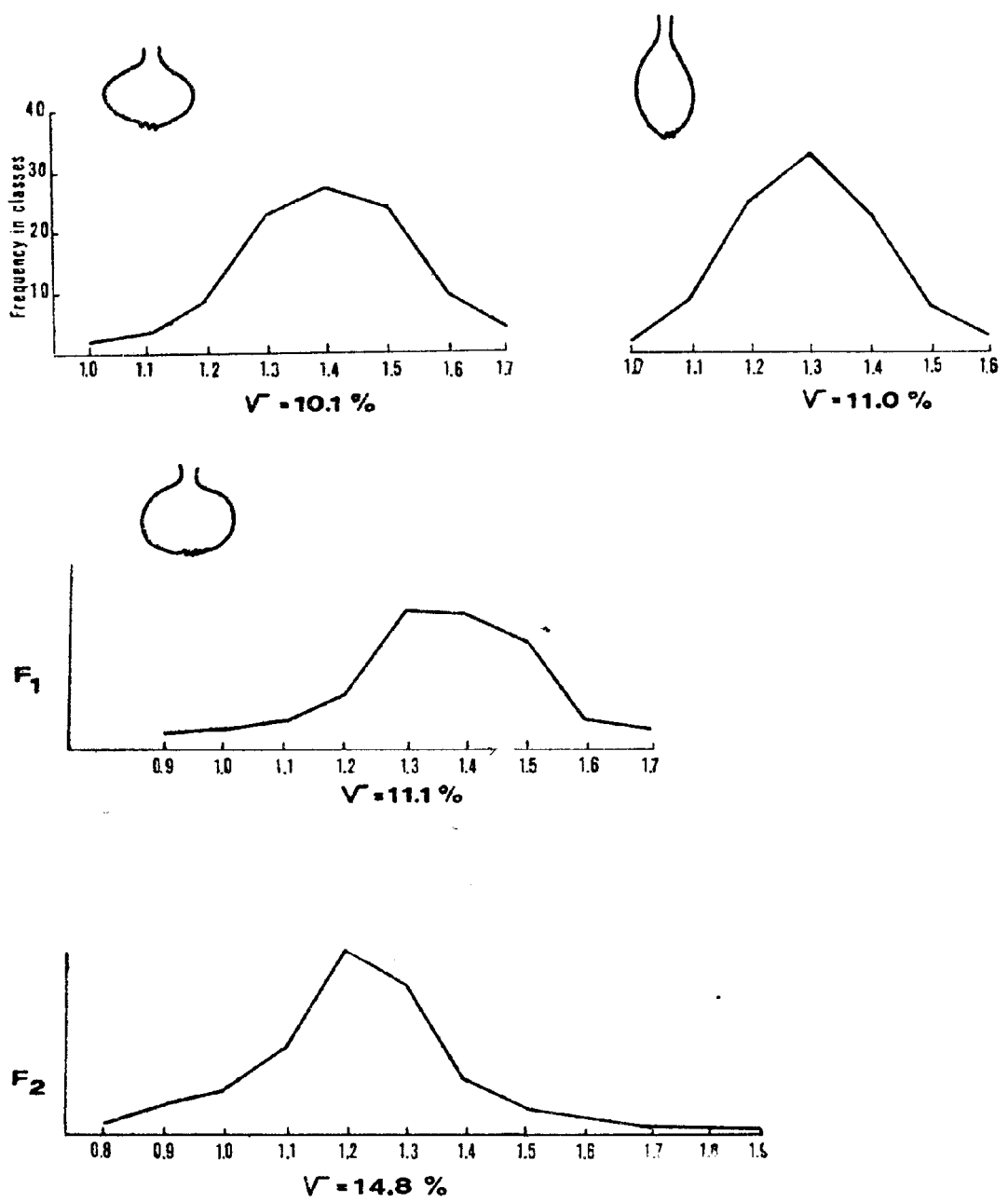

Fig. 8. Histogram of onion root disc diameter of parental forms, $F_{1}$ and $F_{2}$ (A-16 $\times$ C-177). 1977. Classes in $\mathrm{cm}$

each of the two years under study, differed very evidently. The color of the dry skin in $F_{1}$ hybrids was intermediate between that of the parents, that is, lighter than the darker parent. It seems then, that in order to obtain dark dry skin in $F_{1}$ hybrids, both parental components should have dark dry skin.

The variability range of dry skin color in $\mathrm{F}_{2}$ hybrids did not clearly exceed that of $F_{1}$ hybrids.

A rather high heritability was found of very important bulb 
characteristics such as: weight (heritability coefficiert ranged from 51.3 to $71.9 \%$ ), height $(30-80 \%)$, diameter $(50-70 \%)$, dry skin thickness (30$-60 \%$ ), collar shape $(30-50 \%)$, bulb firmness $(32-48 \%)$ (Tables $3-9)$.

The high values of the heritability coefficients of these traits suggest that, for example, variability in $F_{1}$ hybrids was determined to $30-70 \%$ by genetic factors and to $30-50 \%$ by environmental conditions.

The high heritability of the discussed traits indicates a definite possibility of increasing the effectiveness of selection and attaining a high degree of heterosis.

The heritability of the remaining traits, such as dry skin thicness and root disc diameter, was low in most of the hybrids. The coefficients were relatively high only in a few $F_{2}$ hybrids. For this reason, a large number of breeding lines are needed in order to pick out the best of them for improvement of the thicness of the dry skin.

The relatively low heritability coefficients of dry skin thicness and root disc diameter in the studied material were the result of the strong influence of the environment on these traits.

\section{DISCUSSION}

The high variability of the weight, firmness and diameter of onion bulbs of some of the parental lines points to the high combination ability of these lines. Such lines have great meaning for obtaining $F_{1}$ hybrids.

The use of visual evaluation of certain traits, according to the UPOV classification, is easy and not work-consuming, although not very exact. This method can be successfully used in the practical cultivation of onions for the evaluation of some of the characteristics connected with the shape of the onion bulbs, adherence and color of the dry skin.

This method showed that the $\mathrm{C}$ lines used in this study, with the exception of line C-16, were characterized by elliptic or ovate bulbs. Good uniformity and depression, typical for inbred lines, were found in these lines. For this reason, it seems that this shape indicates that they are homozygous to a high degree. The slight variability which was observed was probably due to unfavorable effects of the environment. Some of the lines were not completely homozygous because they were developed by the recurring breeding method, using partial inbreeding. Full inbreeding of these lines was impossible due to the lowering of the viability of the plants, as was observed in earlier studies (Dor u chow ski, 1968).

Lines A exhibited higher shape variability. Their shape was close to spherical. This shape, as has been found during long breeding experience, constantly undergoes segregation to a small degree, in spite 
of partial inbreeding lasting many years. Hence, this bulb shape is unstable and is probably determined by many genes.

The bulb collar in $\mathrm{C}$ lines was thicker than in A lines. This was connected with the typical elongated shape of the $\mathrm{C}$ line bulbs. The position of the root disc in line A bulbs was more varied. It was outstanding and intermediate between outstanding and deep. The line $\mathbf{C}$ paternal lines with elongated bulbs usually had outstanding root discs. They were equalized in respect to this trait, as shown by the low variability coefficient. Until now, the onion breeders mentioned at the beginning of this paper have not studied uniformity of collar shape and root disc.

The variability of the studied onion bulb traits shown on the histograms $(1-7)$ is very similar to the trait variability in maize shown by E a s t $(1908 ; 1936)$ in his classic studies in which he crossed varieties with long and short cobs. In spite of the fact that $E$ as $t$ had inbred lines, he also found deviations in the direction of extreme values in $F_{1}$ hybrids.

Examination of the variability of quantitative characteristics such as weight, height and bulb diameter etc., both in homozygous parental forms and in genetically uniform heterozygous $F_{1}$ populations, did not uncover identical traits, but showed variability to a certain, limited degree, as can be seen on the histograms (Figs. 3, 4, 5). This variability, which was not hereditary, was the result of changing environmental factors. In $F_{2}$, the variability curve expanded and flattened. The variability range oscillated in the region of extreme values (Figs. 3, 4, 5). This variability was the result not only of environmental factors, but also of segregation of probably cumulative genes, influencing quantitative traits. The inheritance of many quantitative traits in the onion is determined by more or less numerous pairs of cumulative genes. When parental forms had the appropriate composition of cumulative genes, the hereditary variability of $F_{2}$ exceeded the range delineated by the parental forms (Figs. $3,4,5$ ). In $F_{2}$, individuals were obtained which surpassed the parental forms in respect to the studied trait. There is the possibility of using them in developing new varieties having more favorable utilitarian traits than the parental forms used in the crosses, for example, height (Fig. 3), diameter (Fig. 4) and thin collar (Fig. 5). The possibility of selecting onions from $F_{2}$ having the desired thin collar (Fig. 5) should be especially emphasized.

$\mathrm{K}$ a m pe (1967) found that such quantitative traits as: weight, height, diameter, shape and storage value react more strongly to environmental factors than do such qualitative traits as dry skin color. Under extremely different environmental conditions, large differences in the variability 
of important bulb characteristics can be found from year to year. For this reason, the presented results are typical for breeding materials under precisely defined conditions. Similar relationships have been found by researchers in the United States (M c C oll u m, 1966; 1968), England (Dowker and Fennell, 1974) and France (Schweisg uth, 1978). Very probably, the effect of gene action in $F_{1}$ was neutralized by the variability of the environment, because the onion is a species exceptionally sensitive to environmental conditions.

Dowker and Fennell (1974) are of the opinion that the results of the studies done until that time showed the sensitivity of all onion varieties to the environment. In addition, these authors believe that limiting the bulb weight variability in the population brings about at the same time the limitation of bulb shape variability.

As can be seen from the data in Tables 3-9, high heritability of many traits in the same $F_{2}$ hybrids was found. These results indicate the possibility of attaining high effectiveness of selection. The heritability of bulb weight in the studied $F_{1}$ hybrids was similar (it ranged from $30-70 \%$ ) to that found by researchers abroad in their native varieties, for example, Nakamura (1959) in var. 'Senshuki' (17-40\%) and M c C oll u m (1966) in var. 'Sweet Spanish Type' (34-68\%). H a n s o n (1963) was of the opinion that heritability is important in breeding when carrying out selection by foreseeing the effectiveness of selection and in programming breeding methods. A high degree of heritability insures quick progress in onion breeding. The traits of the offspring should differ from their parents in respect to variability of onion bulb characteristics. This increases the changes of obtaining hybrids with the desired utilitarian traits.

\section{CONCLUSIONS}

1. The highest variability was exhibited by weight and by dry skin thickness, slightly lower variability by collar thickness, root disc diameter and bulb diameter. The lowest variability was displayed by bulb firmness and height. Low variability and good uniformity of utilitarian traits were found in several parental lines (A-63, C-6, C-141, C-177) which indicates that they are homozygous to a high degree. The parental lines were more sensitive to changes in the environmental conditions than the $F_{1}$ hybrids. The smaller reaction of the $F_{1}$ hybrids to unfavorable environmental conditions emphasizes their high production value.

2. Studies on the variability of such quantitative traits as weight, bulb height and diameter, etc., both in homozygous parental forms and 
in genetically uniform heterozygots in $F_{1}$ generations did not show identical properties, but a small degree of variability. This variability, which was not heritable, was the result of environmental factors. The segregation of traits took place in $F_{2}$, which caused the expansion and flattening of the variability curve. The variability range oscillated in the: region of extreme values. This variability was not only the result of segregation of, probably, cumulative genes influencing the development of quantitative traits, but also the result of environmental influences.

3. The analysis of bulb shape, color and dry skin adherence based on the frequency distributions of traits classified according to UPOV can be very helpful in breeding, due to the differences in variability of studied populations.

4. A relatively high heritability was found of very important utilitarian traits such as: weight (heritability coefficient from 51.3 to $71.9 \%$ ), height $(30-80 \%)$, dimeter $(50-70 \%)$, dry skin thickness $(30-60 \%)$, collar shape $(30-50 \%)$, bulb firmness $(32-48 \%)$. The high heritability of the listed traits indicated a realistic possibility of increasing the effectiveness of selection and achieving a high heterosis of crop yield and improving bulb shape (especially its uniformity).

\section{REFERENCES}

Doruchowski R. W., 1968. Badania nad opracowaniem metody hodowli mieszańców heterozyjnych cebuli (Allium cepa L.) dla polskich warunków. Ph. D. thesis. Instytut Warzywnictwa, Skierniewice.

Dowker B. D., Fennell J. F. M., 1974. Some responses to agronomic treatments of different genotypes of bulb onions, Allium cepa L. J. Hort. Sci. 4: 1-14.

Dowker B. D., Fennell J. F. M., 1974. Heritability of bulb shape in some north European onion varieties. Ann. Appl. Biol. 77: 61-65.

Ea s t E. M., 1908. Inbreeding in corn. Rept. Connecticut Agric. Exp. Sta. for 1907, pp. 419-428.

E a t E. M., 1936. Heterosis. Genetics 21: 375-397.

E 1-Shafie M. W., Ahmed A. A., 1977. Inheritance of earliness and bulb weight in the common onion (Allium cepa L.) Libyan Journal of Agriculture 6: 253-266.

F a l coner D. S., 1960. Introduction to quantitative genetics. Oliver and Boyd, Edinburgh and London, pp. 1-365.

Han s on W. D., 1963. Heritability in statistics and plant breeding. Eds. W. D. Hanson and H. F. Robinson. Publication 982. National Academy of Sciences National Research Council. Washington. D. C., pp. 125-140.

Harada C., 1961. Heterosis of the quantitative characters in the silk worm. Bull. Sericult. Exp. Station. Tokyo. 17.1. 
Kampe F., 1967. Variabilität und Vererbung von wirtchaftlich wichtigen Weteigenschaften bei der Säzwiebel. Archiv für Gartenbau 15: 273-283.

Mahmud I., Kramer H. H., 1951. Segregation for yield, height and maturity follow a soybean cross. Agronomy Journal. 43: 605-609.

M c Col1um G. D., 1966. Heritability and genetic correlation of some onion bulb traits. The Journal of Heredity. May - June. 57: 105-110.

M c Collu m G. D., 1968. Heritability and genetic correlation of soluble solids, bulb size and shape in white sweet spanish onion. Canadian Journal of Genetics and Cytology 10: 508-514.

$\mathrm{Nakamura}$ G. D., 1959. Studies on the breeding of Allium cepa L. Estimating heritability. Japan. J. Breeding 8: 255-260.

$\mathrm{Schw}$ is guth B., 1978. Limites a l'utilisation de toute la variabilite genetique chez l'Oignon (Allium cepa L.) et quelques propositions pour les surmonter. Ann. Amel. Pl. 28: 709-712.

Zmienność i odziedziczalność niektórych cech użytkowych form rodzicielskich, mieszańców $\mathrm{F}_{1}$ i $\mathrm{F}_{2}$ cebuli (Allium cepa L.)

\section{Streszczenie}

Badania przeprowadzono w Zakładzie Hodowli i Genetyki Instytutu Warzywnictwa $w$ Skierniewicach $w$ latach 1977-1978. Przebadano 15 mieszańców $F_{1}, 11$ mieszańców $F_{2}$ oraz 16 komponentów rodzicielskich, czyli 8 linii matecznych (męskosterylnych A) i 8 linii ojcowskich (wsobnych C). Badano zmienność i odziedziczalność następujących cech ilościowych cebuli: masę, wysokość, średnice, grubość suchej łuski, twardość cebul, grubość szyjki liściowej i średnice piętki. Zmienność i odziedziczalność masy i średnicy oraz kształtu szyjki była duża. Duża była również zmienność grubości suchej łuski, natomiast mala byla odziedziczalność tej cechy. Najmniejszą zmiennością i odziedziczalnością charakteryzowały się twarỏość i wysokość cebul. Największego postępu hodowlanego można spodziewać się w polepszeniu masy i ksztaltu cebul (jego wyrównania). 